\title{
Desarrollo de un Programa de Oclusiones Totales Crónicas en la República Argentina - Colegio Argentino de Cardioangiólogos Intervencionistas
}

\author{
Development of a Program of Chronic Total Occlusions in the Argentine \\ Republic - Colegio Argentino de Cardioangiólogos Intervencionistas
}

Coordinador: Lucio Tiburcio Padilla.

Autores: Marcelo Ángel Abud, Alejandro Álvarez Iorio, Miguel Ballarino, Claudio Cigalini, Ignacio Cigalini, Fernando Cohen, José Cruzado, Aníbal Damonte, Alejandro Fernández, Arturo Fernández Murga, Alejandro Goldsmit, Pablo Kantor, Paola Kushnir, Guillermo Migliaro, Gustavo Pedernera, María Paz Ricapito, Agustina Sciancalepore.

Comité Revisor: José Amadeo Álvarez, Daniel Berrocal, Alejandro Cherro, Ricardo A. Costantini, Fernando Cura, José Oscar Gómez Moreno, Hugo Londero, Oscar Mendiz, Alberto Sampaolesi, Ernesto Torresani.

Revista Argentina de Cardioangiología Intervencionista 2018;9(1):15-38. Doi: 10.30567/RACI/201801/0015-0038

\section{PROGRAMA DE OCLUSIONES TOTALES CRÓNICAS. ¿CÓMO INICIARLO?}

La angioplastia a una oclusión total crónica (OTC) es, sin lugar a dudas, el procedimiento más desafiante en el intervencionismo coronario. Los avances tecnológicos que están llevando esta práctica a lugares probablemente impensados en épocas anteriores, sumado al creciente interés de los cardiólogos intervencionistas en el tratamiento de estas lesiones, han aumentado francamente la eficacia de esta intervención en los últimos años.

Sin embargo, la percepción de altas tasas de fracaso y de la alta complejidad del procedimiento frecuentemente impiden que los médicos deriven a los pacientes con OTC al cardiólogo intervencionista ${ }^{1}$.

Para superar esta reticencia, es necesario contar con un programa de OTC que involucre a intervencionistas experimentados para construir habilidades en el procedimiento y demostrar los resultados y la calidad del operador ${ }^{2-6}$. Asimismo, un programa exitoso se acompañará de beneficios financieros permitiendo ayudar a superar algunas de las barreras operativas más significativas para el empleo de estas técnicas.

\footnotetext{
1. Colegio Argentino de Cardioangiólogos Intervencionistas.

$\triangle$ Correspondencia: Dr. Lucio Tiburcio Padilla: Itpadilla@icba.com.ar; Dr. Arturo Fernández Murga: fdezmurga@tucbbs.com.ar

Los autores no declaran conflictos de intereses.

Recibido: 21/12/2017| Aceptado:04/03/2018
}

Por esto, su implementación requiere de una amplia infraestructura constituida por: operadores dedicados que tengan la libertad para desarrollar sus habilidades y aprender nuevas técnicas; laboratorios de cateterismo capaces de brindar los recursos materiales y soportar el tiempo procesal de estos tratamientos complejos; equipos de trabajo que consten de enfermeras/os, técnicos radiólogos, cuidados intensivos, cuidados posteriores al procedimiento y cuestiones periprocesales, como la exposición a la radiación y la dosis de contraste.

Es así que la recanalización de OTC exige habilidades particulares y un equipo especializado, con puntos específicos que se desarrollan a continuación.

\section{OPERADORES}

La primera aproximación debe estar centrada en el paciente. Es decir, la selección de este es fundamental. Cerca del $15-30 \%$ de los pacientes remitidos para coronariografía (CCG) tienen una OTC y muchos de ellos son elegibles para angioplastia. Múltiples variables serán tomadas en cuenta en este momento, como, por ejemplo, el cuadro clínico, edad, características angiográficas, frecuencia de los síntomas y comorbilidades (ver más adelante).

Asimismo, el operador deberá realizar un aprendizaje completo sobre angioplastia a OTC en términos de técnicas, familiarizarse con la nomenclatura y el arsenal de materiales a través de la bibliografía, libros de texto, simuladores, cursos, demostraciones en vivo y la industria.

El obstáculo más significativo para el desarrollo de un 
programa de estas características es la inexperiencia del operador, lo que afecta los resultados y la rentabilidad del procedimiento. Los estudios han demostrado que los operadores necesitan como mínimo 75 procedimientos/año para asegurar resultados de alta calidad y mantener su competencia ${ }^{7,8}$. Además, es necesario contar con experiencia en por lo menos 1000 angioplastias no OTC, manejo de accesos vasculares múltiples y procedimientos complejos (como angioplastia de tronco de la coronaria izquierda no protegido, bifurcaciones, perforaciones y tratamiento de calcificación coronaria).

Es muy importante asistir a cursos dedicados a angioplastia a OTC con casos en vivo. Esto es un gran motivador para iniciar un programa. Los casos son realizados por operadores experimentados, permitiendo la participación interactiva con los participantes y el panel.

Por otra parte, es recomendable contar con la supervisión de un proctor en angioplastia a OTC al inicio de la curva de aprendizaje, especialmente al implementar las más exigentes técnicas de cruce, como la anterógrada por disección/reentrada y la retrógrada; de esta forma se realiza el procedimiento con más seguridad y eficiencia. Por otra parte, ayuda a establecer una red que puede utilizar para apoyo y asesoramiento en casos posteriores. Un papel clave del proctor es ayudar a identificar los peligros específicos del caso, así como la prevención activa de las complicaciones.

El enfoque con dos operadores por institución es el más aceptado porque permite acelerar el aprendizaje, identificar más técnicas alternativas y, por lo tanto, pueden mejorar el éxito primario, así como alternar con el operador principal ya que son procedimientos más largos, en donde el cansancio y la fatiga pueden generar errores. Por otra parte, un segundo operador es muy valioso cuando se producen complicaciones que requieren más de una acción inmediata $^{9-11}$.

Una conferencia de OTC local o club a intervalos regulares, dependiendo del volumen de los casos, debe ser un elemento integral de un programa exitoso.

\section{Sala de cateterismo}

Educar al personal del laboratorio de cateterismo ayuda y contribuye al ámbito adecuado para este tipo de procedimientos, no solo para la resolución más rápida de diferentes casos sino también para vigilar cuidadosamente la exposición a la radiación (anunciando la dosis de radiación de Kerma en aire), monitoreo de anticoagulación (medir tiempo de coagulación activado cada 30 minutos) y la cantidad de contraste empleado ${ }^{12}$.

Todo el personal identificado debe estar debidamente informado sobre los aspectos técnicos del procedimiento, incluyendo terminología, equipos y complicaciones potenciales. Asimismo estará a cargo de la comodidad del paciente y la sedación ya que la duración de las intervenciones es prolongada. Es ideal identificar uno o dos técnicos con interés en OTC que estén motivados en aprender el procedimiento y obtener más experiencia.
El soporte avanzado de imágenes es crucial, con sistemas que tengan una calibración periódica de la calidad de las mismas. Los sistemas digitales ofrecen procesamiento de imagen y protocolos de radiación dedicados (generalmente 7,5 frames/s).

Se recomienda designar "Días OTC" permitiendo el enfoque intensivo e ininterrumpido de estos procedimientos, a menudo complejos, para que no afecte de manera negativa la agenda del día. Sería que los centros cuenten con una sala disponible backup ya que la presencia simultánea de emergencias como infarto agudo de miocardio para angioplastia primaria, dificultaría el normal desarrollo del procedimiento. Es recomendable comenzar lentamente con uno o a lo sumo dos casos programados ${ }^{13}$.

La cirugía cardíaca en el centro es deseable pero no obligatoria. En caso de contar con ella, el operador deberá decidir qué casos de OTC podrían ser los más apropiados para abordarlos. Entre las complicaciones que pueden surgir durante estos procedimientos complejos se encuentran la disección, perforación y ruptura del vaso. Por lo tanto, es mandatorio contar con un plan de acción para tales eventualidades. Formalizar una vía para manejar tales complicaciones, además de tener fácilmente disponibles las herramientas básicas para su resolución como kit de pericardiocentesis, stents recubiertos y dispositivos de embolización (ver más adelante).

\section{Administración}

El apoyo de la administración de la institución es clave para el éxito de un programa de angioplastia a OTC, especialmente en la etapa temprana de la curva de aprendizaje, cuando las tasas de éxito no pueden ser tan altas como serán más tarde $e^{14}$.

Se trata en principio de procedimientos prolongados, con utilización de materiales dedicados, algunos de costo incrementado y que requieren de una mayor utilización de la sala que procedimientos no OTC. Sin embargo, es de fundamental importancia resaltar que si una institución es buena en angioplastia a OTC, también será buena en todos los demás procedimientos coronarios. Además, cuando una institución obtiene reconocimiento por hacer angioplastia a OTC, las derivaciones de casos complejos no OTC también aumentan.

\section{Los médicos y pacientes}

No es infrecuente que cardiólogos clínicos e incluso algunos intervencionistas puedan tener una percepción negativa con respecto a la viabilidad, el riesgo potencial de complicaciones y los beneficios de la angioplastia a OTC. La educación sobre el procedimiento y la técnica se dará a lo largo del camino. Asimismo, es importante educar a los pacientes sobre los riesgos y beneficios del procedimiento.

Una planificación cuidadosa, metódica, aprendizaje continuo e implementación de etapas son claves para el éxito ${ }^{15}$. 


\section{CÓMO JUSTIFICAR LA REVASCULARIZACIÓN DE OCLUSIONES TOTALES CRÓNICAS}

\section{Definición}

Las OTC se definen como aquellas lesiones en las que se documenta angiográficamente la interrupción del flujo coronario anterógrado (TIMI 0) de $\geq 3$ meses de evolución o aquellas oclusiones de reciente diagnóstico no atribuibles a eventos isquémicos recientes. Aquellas lesiones con flujo TIMI I se definen como oclusiones subtotales $o$ funcionales, mientras que aquellas lesiones con luz residual pero con ausencia de flujo anterógrado por flujo competitivo proveniente de colaterales se denominan pseudooclusiones. Es importante destacar que en la práctica cotidiana podría resultar complejo diferenciar oclusiones funcionales y pseudooclusiones de una OTC verdadera. Una estrategia ampliamente recomendada para definir con precisión las características de la lesión es realizar una inyección dual por vía anterógrada y retrógrada con el fin de obtener una interpretación más completa de la misma.

\section{Epidemiología}

La prevalencia de OTC varía según diferentes series, reportándose en el 18-52\% de las angiografías diagnósti$\operatorname{cas}^{4,16}$. Sin embargo, en la actualidad la mayor parte de las instituciones solo realizan angioplastia en estas lesiones en el 5-22\% de los $\operatorname{casos}^{17}$, existiendo una marcada variabilidad entre diferentes grupos de intervencionistas (entre el 6-9\% en Estados Unidos y hasta el 60\% en Japón ${ }^{18}$. Esto, a pesar de que en manos expertas la tasa de éxito alcanza el $90 \%{ }^{16,15,19}$. En la actualidad, la estrategia elegida más frecuentemente para el tratamiento de las OTC continúa siendo la cirugía de revascularización miocárdica, mientras que la angioplastia se reserva para los casos en los que la OTC es la única lesión o en pacientes con enfermedad de múltiples vasos simple $e^{20}$.

\section{Beneficios de la angioplastia a oclusiones totales crónicas}

En el año 2006, Werner y cols. publicaron un trabajo emblemático en el que demostraron que aun en presencia de circulación colateral, el lecho distal a las OTC presenta isquemia independientemente del grado de desarrollo de las colaterales $^{21}$. Se ha observado que la revascularización de estas lesiones mejora la carga isquémica de estos pacientes $^{22}$, aunque debe destacarse la marcada heterogeneidad en los métodos de estudio empleados, así como la falta de estudios randomizados que hayan demostrado impacto de la angioplastia en puntos finales duros ${ }^{16}$.

El principal objetivo de la angioplastia a OTC es el alivio de los síntomas y subsecuentemente de la calidad de vida del paciente. El impacto que tiene la intervención en el pronóstico de estos es motivo de controversia en la actualidad. Por ello, la adecuada selección del paciente es fundamental en la obtención de resultados adecuados, particularmente aquellos con síntomas persistentes (considerar la presencia de síntomas atípicos) a pesar del tratamiento médico óptimo (TMO). Aquellos pacientes con evidencia de isquemia extensa en cámara gamma tienen un peor pronóstico y podrían beneficiarse de la intervención ${ }^{23}$. En función de este último criterio, considerar la angioplastia en pacientes asintomáticos con isquemia significativa en pruebas funcionales.

Un metaanálisis de 6 estudios $(\mathrm{n}=1030)$ publicado en 2010 demostró que en pacientes bajo TMO, la revascularización exitosa de la OTC mejoró significativamente el estado anginoso $^{24}$. El estudio TOAST GISE incluyó 376 pacientes con OTC. Aquellos con angioplastia exitosa estuvieron más frecuentemente libres de angina ( 89 vs. $75 \%$; $\mathrm{p}=0,008)$ y tuvieron una prueba de esfuerzo negativa para isquemia ( 73 vs. $43 \%$; $\mathrm{p}=0,001)$ a 1 año de seguimiento, comparados con los que tuvieron una ATC fallida ${ }^{2}$. En el estudio FACTOR $(n=125)$ se demostró que desde el primer mes se evidencia una mejora de los episodios anginosos y de la calidad de vida en los aquellos los pacientes con angioplastia exitosa ${ }^{25}$.

En el estudio OPEN-CTO, en el que se incluyeron 1000 pacientes tratados con angioplastia a OTC utilizando el abordaje híbrido, encontraron que la tasa de éxito del procedimiento ha sido alta (86\%), impactando positivamente en la calidad de vida de los pacientes con mejoría de la disnea e, incluso, en los niveles de depresión ${ }^{26}$.

Recientemente se conocieron los resultados de dos estudios randomizados de amplia relevancia, los cuales aún no han sido publicados formalmente en la literatura que compararon la angioplastia a OTC y el TMO.

En el primero de ellos, el estudio EURO-CTO ( $A$ Randomized Multicenter Trial to Evaluate the Utilization of Revascularization or Optimal Medical Therapy for the Treatment of Chronic Total Coronary Occlusions), se randomizaron 259 pacientes a angioplastia de OTC vs. 137 pacientes asignados a TMO. El punto final primario de eficacia fue el estado de salud a 12 meses y el de seguridad fue el combinado de muerte e infarto no fatal a 36 meses. Con respecto a este último no se observaron diferencias significativas. Con respecto a la revascularización guiada por isquemia fue numéricamente mayor en el grupo TMO aunque no se alcanzó la significación estadística (TMO $6.7 \%$ vs. ATC OTC 2.9\%; $\mathrm{p}=0,10)$. Con respecto al end point de seguridad, los pacientes tratados con angioplastia tuvieron una marcada mejora en la limitación física $(p=0,022)$, en la frecuencia de angina $(p=0,009)$ y en la calidad de vida ( $\mathrm{p}=0,049)$.

Por su parte el estudio DECISIÓN-CTO realizado en Corea randomizó 834 pacientes a angioplastia de OTC vs. TMO. Como punto final se evaluó mortalidad, infarto no fatal, accidente cerebrovascular y necesidad de nuevas revascularizaciones. A pesar de que su diseño tuvo grandes críticas (sesgo de selección, falta de potencia, cruzamiento significativo), no se alcanzó un resultado significativo en favor de la angioplastia coronaria.

Otro efecto reportado de la angioplastia a OTC es la mejora de la función ventricular. Un metaanálisis de 34 estudios ( $\mathrm{n}=2243)$ demostró un aumento significativo de 
la fracción de eyección de 4,44\% (IC95\%: 3,52-5,35; $\mathrm{p}<0,01)$ luego de una angioplastia exitosa ${ }^{27}$. Recientemente se publicaron los resultados del estudio EXPLORE en el que 304 pacientes con Infarto agudo con elevación del segmento ST y la presencia concomitante de una CTO fueron randomizados a angioplastia de OTC o a tratamiento médico posangioplastia primaria. A los cuatro meses, el volumen de fin de diástole y la fracción de eyección evaluados por resonancia magnética nuclear fueron similares en ambos grupos, aunque se observó un marcada mejoría de la Fey en pacientes que recibieron una angioplastia a OTC de la arteria descendente anterior $(\Delta=6,8$; IC95\%: $1,1-12,6 ; p=0,002)^{28}$. Resultados similares se observaron el estudio COREA-AMI ${ }^{29}$.

Como se mencionó previamente, el efecto de la angioplastia a OTC sobre la mortalidad es controvertida. Evidencia proveniente de estudios observacionales y metaanálisis que compararon la angioplastia exitosa de la OTC $v s$. revascularización fallida se ha reportado una disminución de la mortalidad con un odds ratio (OR) de 0,52 (IC95\%: 0,43-0,62; $\mathrm{p}<0,01)^{27}$. En la base de datos del Reino Unido, que analizó 14.000 angioplastias de OTC, la ATP exitosa estuvo vinculada a una mejoría de la sobrevida con un hazard ratio (HR) de 0,72 (IC95\%: 0,62-0,85; $\mathrm{p}<0,0001)^{30}$. Por su parte, un registro japonés de $1424 \mathrm{pa}-$ cientes reportó una disminución de la mortalidad cuando la recanalización estaba asociada a la arteria descendente anterior y la arteria coronaria derecha pero no en la arteria circunfleja ${ }^{31}$.

\section{Recomendaciones de las guías en la revasculariza- ción de oclusiones totales crónicas}

En el año 2009, las sociedades americanas de cardiología publicaron una guía de criterios de uso apropiado en la toma de decisiones en la revascularización de los pacientes con enfermedad coronaria, las cuales fueron actualizadas en el año 2012. Estas recomendaciones toman en cuenta múltiples escenarios incluyendo, los síntomas del paciente, la presentación clínica, el perfil del riesgo, la presencia de TMO y las características angiográficas de las lesiones, entre ellas, la presencia de OTC. En general la revascularización de una OTC es apropiada en pacientes de riesgo intermedio-alto, quienes persisten sintomáticos a pesar del $\mathrm{TMO}^{32}$. Por su parte, las guías europeas le dan a la angioplastia de una OTC una recomendación de clase IIa nivel de evidencia $\mathrm{B}$, teniendo en cuenta las características clínicas del paciente, la factibilidad anatómica y la experiencia del operador $^{33}$.

Como resultado de la gran divergencia que existe entre la postura de las guías societarias más importantes y la experiencia en la práctica cotidiana, el manejo de pacientes con OTC continúa siendo muy heterogéneo entre las diferentes instituciones a lo largo del mundo.

Consideramos que la decisión de efectuar una angioplastia a una OTC deberá centralizarse en el paciente, su condición clínica y las capacidades del centro interviniente con el único objetivo de mejorar la calidad de vida del mismo.

\section{DESCRIPCIÓN DEL ALGORITMO HÍBRIDO EN EL MANEJO DE OCLUSIONES TOTALES CRÓNICAS}

En el año 2012, Brilakis y cols. desarrollaron un algoritmo para cruzar las OTC en forma segura, efectiva y eficiente, empleando todas las técnicas disponibles ajustadas a cada caso en particular, denominado abordaje híbrido ${ }^{13}$ (Figura 1).

La introducción del mismo ha modificado la manera de pensar la angioplastia a OTC por parte de los cardiólogos intervencionistas. Este proceso "mental" abarca cada paso del procedimiento, desde la selección de los accesos, el tamaño del introductor, el catéter guía adecuado para lograr un buen soporte, la necesidad de dispositivos para mejorar el soporte, la guía coronaria inicial en función de las características angiográficas de la placa, definir las colaterales aptas para un eventual abordaje retrógrado, entre otras variables. Actualmente contamos con tres técnicas específicas para cruzar una OTC: vía anterógrada con escalonamiento de guías coronarias, vía anterógrada con estrategia de disección/reentrada y la vía retrógrada ${ }^{34}$, y son la base de las diferentes alternativas propuestas en el algoritmo.

Todo esto ha permitido optimizar el tiempo del procedimiento así como las dosis de radiación y de contraste empleados. Así también, exige que el operador sea un experto en el manejo de las diferentes técnicas que son necesarias para realizar ATC para poder recorrer el algoritmo en su totalidad.

\section{Primer paso: doble inyección}

El uso de la doble inyección es el primer paso para abordar este algoritmo y constituye una condición esencial para llevarlo a cabo. La doble inyección permite obtener una definición angiográfica de la lesión más clara, lo que resulta fundamental para la toma adecuada de decisiones y así aumentar el éxito de la intervención manteniendo un adecuado perfil de seguridad ${ }^{35}$.

Los objetivos principales de esta técnica son:

- Mejorar la caracterización del cap proximal y distal.

- Definición precisa de la longitud de la lesión.

- Reconocer el tamaño del vaso distal a la oclusión.

- Identificar la presencia de bifurcaciones distales a la oclusión.

- Evaluación de la circulación colateral.

- Identificar la localización de la guía coronaria en el intento de cruzar la lesión.

La técnica de doble inyección recomendada por los expertos es inyección inicial en el vaso donante y luego de 2-3 segundos una inyección en la arteria diana, en una proyección que permita la entera visualización de la anatomía coronaria $^{34}$.

\section{Segundo paso: evaluación de la anatomía de la OTC}

El siguiente paso se basa en la evaluación exhaustiva de la lesión, lo cual debería ser llevado a cabo por todo el 


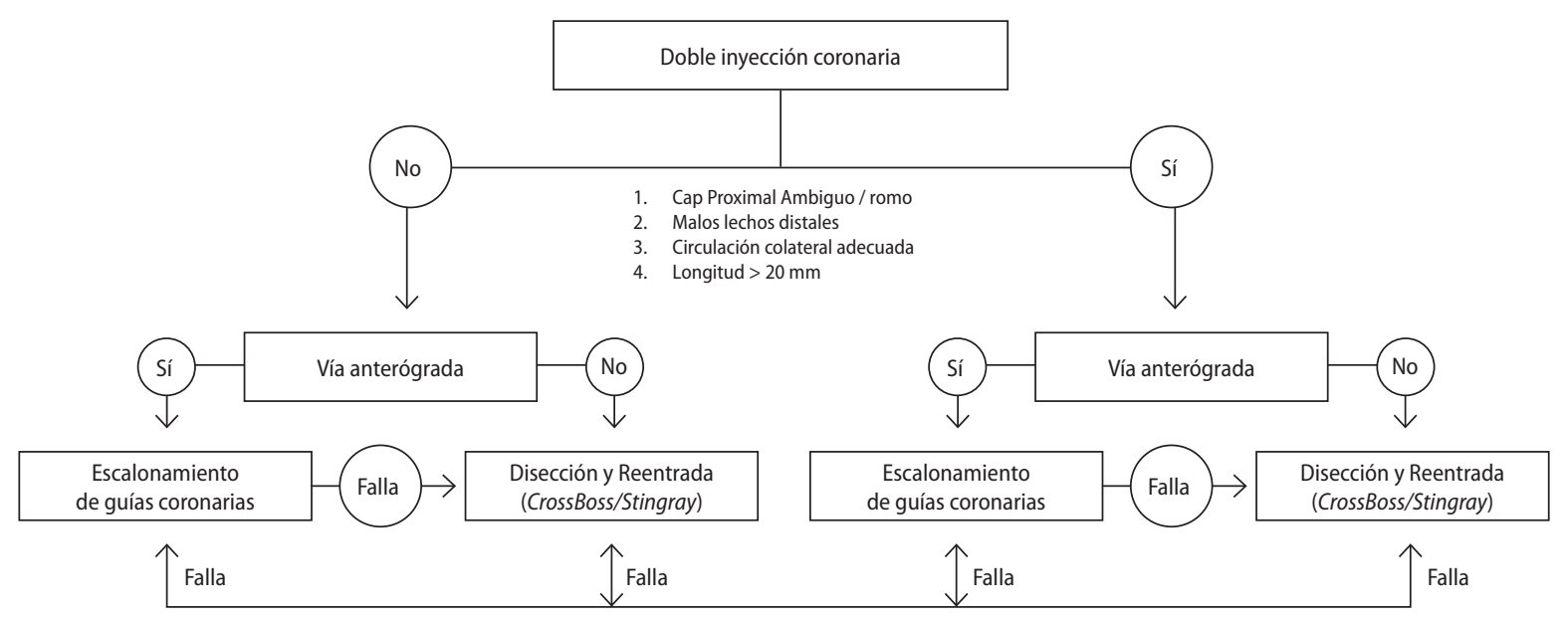

Figura 1. Descripción del algoritmo híbrido.

equipo de intervencionistas, técnicos y enfermeros participantes, para que todo el equipo conozca la estrategia de antemano. Se recomienda dedicar al menos 20-30 minutos para identificar la anatomía de la OTC y planificar adecuadamente la estrategia. Por esta razón es que se sugiere no realizar una angioplastia en OTC ad hoc.

La importancia de este segundo paso radica en que, de acuerdo a la morfología de la lesión y sus diferentes características, elegiremos una u otra técnica de cruce, así como nos inclinaremos por el uso de determinados materiales dedicados para cada caso en particular.

\section{Morfología del cap proximal}

Definir la morfología del cap proximal tiene una influencia fundamental en la elección de la técnica de cruce, particularmente en evaluar la factibilidad de la vía anterógrada. En líneas generales se definen tres morfologías:

- Romo, conocido en la literatura anglosajona como blunt stump.

- Cónico, conocido en la literatura anglosajona como tapered tip.

- Ambiguo, es decir que no es bien definido el curso subsiguiente del vaso. La principal causa de "ambigüedad" es la presencia de ramas laterales o colaterales en puente a nivel proximal.

La presencia de blunt stump o de un cap ambiguo son predictores de ATC no exitosa para la vía anterógrada. En el primer caso, el estudio J-CTO encontró que aumentaba entre 2-3 veces la probabilidad de ATC fallida ${ }^{36}$. En el segundo caso, la presencia de un cap ambiguo motivó el empleo de técnicas de disección/reentrada anterógrada en el estudio RECHARGE ${ }^{37}$ mientras que los expertos recomiendan acceder por vía retrógrada como estrategia inicial.

Por su parte, la presencia de tapered tip suele asociarse a lesiones con menor contenido de colágeno y los microcanales suelen disponerse en posición central, en clara relación con la luz real del vaso. En este contexto, el abordaje anterógrado debería ser la primera opción.
Como se mencionó recientemente, la definición del cap proximal tiene una clara correlación con la histología de la lesión. Esto ha sido ampliamente estudiado por diferentes técnicas: necropsias, tomografía computada intravascular y ultrasonido intravascular. Un aspecto histopatológico destacable es la preservación de la arquitectura vascular en la que la túnica íntima (en conjunto con la placa) puede distinguirse adecuadamente de la túnica media y la túnica adventicia. Incluso la lámina elástica externa suele encontrarse intacta. Esto permite la revascularización percutánea mediante técnicas de disección y reentrada.

En el trabajo de Katsuragawa y cols. realizaron autopsias a 10 corazones con OTC $^{38}$. Los segmentos ocluidos fueron cortados en láminas de $10 \mu \mathrm{m}$ de espesor. Como hallazgos de relevancia destacamos que a nivel del cap proximal se evidencia la mayor densidad de placa y la mayor evidencia de remodelado vascular. Por otra parte, las lesiones tipo tapered tip presentaron un mayor porcentaje de tejido conectivo no compacto, con microcanales que suelen atravesar por el centro de la oclusión hasta en el $80 \%$ de los casos y suelen tratarse de trayectos más cortos. Contrariamente, las lesiones tipo blunt stump presentan un mayor porcentaje de tejido fibroso en su constitución, no presentan microcanales que atraviesan la lesión, sino que se dirigen hacia el espacio subintimal.

Por su parte, los trabajos de Srivatsa y cols. demostraron que las oclusiones jóvenes (entre 3 meses y un año) presentan una composición predominante de colesterol y células espumosas, mientras que el componente fibrocálcico aumenta con la edad de la lesión ${ }^{39}$. Otros investigadores encontraron que cuanto más definido es el blunt stump más crónica es la oclusión ${ }^{40}$.

Remarcamos estas diferencias en las características histológicas de la lesión para enfatizar la importancia de comprender correctamente la anatomía angiográfica y como la obtención de información exhaustiva contribuye en la toma de decisiones. Las lesiones de punta cónica presentan tejido menos compactado, son más "blandas", presentan más frecuentemente recanalización central y suelen ser más cortas. Esto explica por qué es factible emplear la 
vía anterógrada para cruzar este tipo de lesiones con altas probabilidades de éxito. Por otra parte, las lesiones con un cap romo tienden a presentar tejido conectivo más compactado, con mayor calcificación, lo que hace que estas lesiones sean más "resistentes" y adicionalmente suelen ser de mayor longitud. En estos casos, cruzar la lesión por vía anterógrada es menos factible, por lo que ante la presencia de este tipo de lesiones el operador deberá planificar de antemano el uso potencial de una estrategia de disección o reentrada o directamente por vía retrógrada. A su vez, esto es de fundamental importancia para la elección de las guías coronarias. En lesiones tipo blunt stump será recomendable emplear guías coronarias con mayor fuerza de penetración desde el inicio.

Es importante que los operadores consideren realizar proyecciones no convencionales en caso de que el inicio de la lesión no esté adecuadamente definido, logrando evaluar el cap proximal en al menos 3 proyecciones ortogonales fijas. Si de este modo no se logra una adecuada visualización, podría colocarse un microcatéter proximal al cap (no en el cap) e inyectar, preferentemente con jeringa de 3 cc. En algunos casos la CCG puede resultar insuficiente para cumplir con este requisito, por lo que complementar con información proveniente de imágenes intravasculares es una opción recomendable o incluso realizar una angiotomografía coronaria multicorte.

\section{Longitud de la lesión}

Cuanto más larga es la oclusión, menor es la probabilidad de cruzar la lesión en forma exitosa. El punto de corte empleado actualmente es de $20 \mathrm{~mm}$ y tanto en el estudio J-CTO como en el estudio RECHARGE este valor se asoció significativamente a ATC fallida ${ }^{36,37}$ además de que aumenta las posibilidades de cruzar inadvertidamente por el espacio subintimal cuando se avanza por vía anterógrada $a^{41}$. Por esto, la presencia de oclusiones de longitud significativa deberá sugerirle al operador la necesidad potencial de emplear técnicas de disección y reentrada o abordaje por vía retrógrada, dado que la probabilidad de cruce por vía anterógrada con escalonamiento de guías coronarias es menor, más aún si se asocia a tortuosidad o calcificación severas.

La longitud de la lesión será una medida de la dificultad del procedimiento y la duración del mismo. Siguiendo con el concepto del abordaje híbrido, no es aconsejable disponer de largos períodos de tiempo intentando cruzar con técnica anterógrada, dado que, los tiempos de intervención podrían prolongarse significativamente y además la disección y la generación de un hematoma en la pared de la arteria podrían condicionar eventualmente la factibilidad de las siguientes técnicas, además de aumentar el riesgo de perforación ${ }^{42}$.

Como se mencionó recientemente, la inyección dual es fundamental para determinar la longitud real de la lesión durante el diagnóstico. En caso de que no quede clara la longitud de la lesión ni las características de la misma, sugerimos realizar una angiotomografía coronaria multicorte (ver más adelante).

\section{Caracteristicas del vaso distal a la oclusión}

Dos aspectos relevantes son tamaño del vaso distal a la oclusión, así como la presencia de una bifurcación cercana al cap distal. En el primer caso, cuando el vaso es de fino calibre, existe una posibilidad elevada de cruzar la lesión por falsa luz cuando se accede por vía anterógrada por lo que en estos casos acceder por vía retrógrada en primera instancia sería recomendable. En el segundo caso, frente a la presencia de una bifurcación cercana al extremo distal sería conveniente evitar la técnica de disección y reentrada por el alto riesgo de ocluir la rama latera ${ }^{43}$.

Para la evaluación del vaso distal a la oclusión es fundamental realizar inyecciones fuertes por vía contralateral, con adquisiciones prolongadas para que se llene adecuadamente este segmento del vaso.

\section{Circulación colateral}

La valoración adecuada de la circulación colateral es altamente relevante en la planificación de la intervención dado que representa el camino a seguir durante el abordaje retrógrado. La misma deberá incluir el tipo de circulación (septal, epicárdica, by-pass), el tamaño del vaso, la tortuosidad, el ángulo con el vaso donante y el sitio por el cual ingresa a la arteria que se desea tratar.

Los vasos colaterales se definen como aquellos que conectan un territorio suplido por una arteria coronaria epicárdica con otro territorio suplido por otra arteria coronaria ${ }^{44}$. De este modo representan una fuente alternativa de irrigación en territorios miocárdicos amenazados por la presencia de una oclusión en el vaso fuente. Trabajos en animales demostraron que, si bien este bypass natural podría ser suficiente en la irrigación en el reposo, no suele satisfacer la demanda de irrigación durante el esfuer$\mathrm{zo}^{45}$. En el contexto de OTC ya se ha comentado previamente la experiencia de Werner y col utilizando doppler intracoronario.

Para cuantificar la circulación colateral existen numerosos métodos. El más sencillo es mediante angiografía y fue propuesto por Rentrop y col en el año 1985 [46].

- Grado 0: no se visualiza circulación colateral.

- Grado 1: la circulación colateral llena ramas laterales de la arteria pero el contraste no alcanza la arteria epicárdica.

- Grado 2: la circulación colateral llena parcialmente la arteria epicárdica.

- Grado 3: la circulación colateral llena completamente la arteria epicárdica.

Esta escala tiene importantes limitaciones: en primer lugar no es una medida objetiva del flujo a través de las colaterales. Además, es dependiente de la presión arterial, de la fuerza de inyección y de la duración de la adquisición.

Otra escala empleada es la de Werner ${ }^{47}$ que establece la circulación colateral de la siguiente manera:

- Grado 0: ausencia de conexión continua.

- Grado 1: conexión continua tipo filiforme.

- Grado 2: conexión continua tipo rama lateral pequeña. 
Para cuantificar con mayor precisión la circulación colateral podría aplicarse el indice de fujo por colaterales, el cual puede obtenerse por medio de mediciones de velocidad del doppler intravascular o por mediciones de presión. Un valor $>0.30$ ha sido considerado como suficiente para mantener una irrigación adecuada en reposo, aunque no ha sido rigurosamente validado.

Finalmente, la realización de un electrocardiograma (ECG) intracoronario es otra metodología empleada, principalmente en investigación básica. Los tres métodos mencionados (Rentrop, índice de flujo y ECG intracoronario) han sido predictores de eventos adversos. Un metaanálisis publicado en el año $2012(\mathrm{n}=6529)$ encontró que en pacientes con oclusiones agudas, subagudas y crónicas la presencia de circulación colateral se asoció significativamente a menor mortalidad en el seguimiento ${ }^{48}$.

A los fines de este documento, nos referiremos a las colaterales que son aptas para ser intervenidas, es decir, capaces de aceptar el material dedicado al cruce retrógrado: cuerdas, microcatéteres y balones. Desde ya que la colateral utilizada y las dificultades para acceder a la misma variarán según los operadores y el escenario clínico.

Podemos definir cuatro "vías" de conexión entre arterias coronarias nativas:

- Vía de septales: conectan la arteria descendente anterior con la descendente posterior de la coronaria derecha o circunfleja. Son las más utilizadas para el acceso retrógrado en OTC. Una gran ventaja técnica es que como transcurren por el septum interventricular, la ruptura de las mismas raramente predispone a un alto riesgo de taponamiento cardíaco.

- Vía epicárdica desde el ápex: se trata de la conexión entre la arteria descendente anterior y la descendente posterior de la coronaria derecha o circunfleja por medio de las arterias apicales. Se trata, por lo general, de ramos muy tortuosos, tendientes a torcerse con el paso de guías y microcatéteres y provocar isquemia. No suelen utilizarse para el acceso retrógrado.

- Vía epicárdica desde el surco auriculoventricular: sirven de conexión entre la circunfleja y la descendente posterior de la coronaria derecha. En general, son vasos muy finos y tortuosos con riesgo de ruptura. No se recomienda utilizarlos en el abordaje retrógrado.

\section{- Vía a través de puentes quirúrgicos.}

Siguiendo las definiciones de McEntegart y cols., definiremos los siguientes términos para describir las propiedades de vasos colaterales ${ }^{49}$ :

- Tortuosidad de colaterales: presencia de $\geq 2$ curvaturas significativas $\left(>180^{\circ}\right)$ sucesivas $(<2 \mathrm{~mm})$ en colaterales epicárdicas y $\geq 1$ curvatura significativa en colaterales septales que no se desenrollen en diástole que ocurren en una longitud de la arteria $<3 \times$ el diámetro de la colateral.

- Tamaño de la arteria: se define como colaterales pequeñas aquellas $<1 \mathrm{~mm}$ dado que no permiten la navegación de los materiales.
- Entrada a la colateral: se define como una entrada adversa cuando el ángulo de entrada es $<45^{\circ}$ o cuando hay enfermedad o un stent implantado previamente asociados.

- Salida de la colateral: se define como una salida adversa cuando el ángulo de entrada es $<45^{\circ}$ o cuando hay enfermedad o un stent implantado previamente asociados.

- Colaterales de alto riesgo: vasos epicárdicos $\leq \mathrm{a}$ la mitad del diámetro del microcatéter.

En las oclusiones de la arteria coronaria derecha se han descripto alrededor de 20 patrones de circulación colateral. Los más frecuentes fueron ${ }^{49}$ :

- Desde la arteria DA a la DPCD por vía de septales en el $72 \%$.

- Desde la arteria Cx a la PVCD por vía de vasos epicárdicos del surco AV en el $46.9 \%$.

- Colaterales en puente en el 19.3\%.

- Desde la arteria DA a la DPCD por vía de colateral epicárdicas apicales en el 15.4\%.

- Desde la arteria DA a una rama de la CD en el 11,6\%.

- Desde la arteria auricular de la CD a la CD distal en el 9,1\%.

- Desde la obtusa marginal de la CD a la DPCD en el $8,4 \%$

Cuando la OTC se encuentra en la arteria descendente anterior se han descripto 13 conexiones colaterales, siendo las más frecuentes ${ }^{49}$ :

- Desde la DPCD a la DA vía de septales en el 52,3\%.

- Desde una rama de la CD a la DA por vía epicárdica en el 26,8\%.

- Desde la obtusa marginal de la CD a un ramo DG en el $22,9 \%$.

- Desde un ramo DG a la DA distal en el 20,9\%.

- Desde la arteria auricular de la CD a la DA en el $17,6 \%$.

- Desde un ramo septal de DA hacia otro ramo septal de DA distal o apical en el 15,7\%.

Finalmente, cuando la OTC se presenta en la arteria circunfleja se han descripto 12 vías colaterales; destacando las más frecuentes, hallamos ${ }^{49}$ :

- Desde un ramo DG a un ramo Mx en el 32,9\%.

- Desde un ramo PV al ramo AV de la Cx en el 20,7\%.

- Colaterales en puente en el $18,4 \%$.

- Desde un ramo Mx hacia otro ramo Mx (autocolaterales) en el 10,5\%.

- Desde el ramo DPCD hacia un ramo Mx en el 9,2\%.

- Desde el ramo auricular de la CD a un ramo Mx en el $8 \%$.

Es importante evitar, de ser posible las colaterales epicárdicas por mayor riesgo de perforación, como así también los vasos severamente tortuosos y/o con enfermedad severa. La preferencia por la vía septal queda de manifiesto en 
el trabajo de Sianos y cols. que involucraron 7 centros europeos especializados en abordaje retrógrado de OTC que en el 79,4\% de los casos se utilizó la vía septal y en los restantes alguna vía epicárdica ${ }^{50}$, mientras que en la serie de Rathore y cols. la vía de septales se empleó en el $67,5 \%$ de los $\operatorname{casos}^{51}$. En el trabajo de McEntegart identificaron que en el $63,5 \%$ de las OTC se observaron colaterales que podrían utilizarse en el abordaje retrógrado ${ }^{49}$.

Recomendamos una evaluación exhaustiva de la circulación colateral mediante múltiples proyecciones para lograr desplegar adecuadamente el trayecto de las mismas, como así también para evaluar correctamente el sitio de unión con el vaso a tratar. Se ha descripto que colaterales de grado $\geq 1$, tortuosidad del vaso colateral $<90^{\circ}$ y un ángulo de conexión entre la colateral y el vaso receptor $<90^{\circ}$ fueron predictores independientes de éxito del cruce por vía retrógrado ${ }^{51}$.

Un aspecto técnico relevante es seleccionar la colateral que llegue a la arteria diana a una distancia importante del cap distal para permitir que la guía coronaria y el microcatéter se presenten adecuadamente y con buen soporte frente al mismo.

La presencia de circulación colateral bien desarrollada debería sugerirle al operador rotar al abordaje retrógrado con mayor antelación o incluso utilizarlo como primera opción para llevar adelante un procedimiento eficiente.

\section{Tercer paso: decidir la estrategia inicial}

El tercer paso del algoritmo es definir la técnica de cruce inicial, en función de la morfología de la lesión evaluada tomando en cuenta la composición de la placa, la anatomía del paciente y fundamentalmente las características de la lesión.

En resumen, el abordaje anterógrado se prefiere como estrategia inicial en lesiones cortas $(<20 \mathrm{~mm})$, con cap proximal bien definido, principalmente tipo cónico y cuando no hay calcificación severa. La vía anterógrada con escalamiento de cuerdas es la estrategia inicial en la gran mayoría de los casos. La guía coronaria inicial podría variar en función de las preferencias del operador y las características de la lesión. El concepto de escalonamiento de guías coronarias se refiere a comenzar con cuerdas de punta más suave, progresando en la rigidez de las mismas tras un intento fallido de cruzar con la seleccionada inicialmente. En cualquier caso, se recomienda el uso de microcatéter para mejorar el soporte y no perder la posición en el cambio de las mismas.

La estrategia de disección y reentrada es una alternativa muy útil, particularmente en lesiones largas (>20 mm) o ante la presencia de un cap proximal complejo. En líneas generales se recomienda el uso de técnicas controladas que generen disecciones limitadas para disminuir el riesgo de ocluir un ramo lateral, disminuir la longitud de stents implantados y la probabilidad de reestenosis ${ }^{52}$. La técnica STAR (subintimal tracking and re-entry) no está recomendada dado que se asocia a mayores tasas de reestenosis ${ }^{53}$.

Por su parte, la vía retrógrada ha sido en gran medida res- ponsable del aumento en la tasa de éxito global de la intervención. Este abordaje se prefiere en lesiones con blunt stump/cap proximal ambiguo, severamente calcificadas, con malos lechos distales, ante la presencia de buenos vasos colaterales y, dado que se utiliza una menor cantidad de contraste, en pacientes con insuficiencia renal ${ }^{34}$.

Uno de los conceptos más importantes introducidos por del abordaje hibrido es el de rotar rápidamente de una técnica de cruce a otra cuando la estrategia inicial no nos permite cruzar la lesión en un tiempo razonable ${ }^{13}$. La concepción con la que abordar estas lesiones debería basarse en un proceso dinámico, que nos permita avanzar a diferentes etapas en forma expeditiva evitando bajo todos los medios posibles la inducción de injuria en la pared arterial que dificulte aún más el procedimiento. Esto suele ser frecuente de ver en lesiones severamente calcificadas y largas, en las que el paso de la cuerda a través de una falsa luz puede inducir la formación de hematoma de la pared bloqueando las potenciales vías de cruce.

Otro aspecto de fundamental importancia en la planificación de la intervención y en la toma de decisiones terapéuticas es la posibilidad de predecir el éxito del procedimiento. Con esta finalidad, el sistema de puntuación más empleado actualmente es el J-CTO. Brevemente, fue introducido en el año 2011 por Morino y cols. enfatizando en la probabilidad de cruzar la lesión por vía anterógrada en $\leq 30$ minutos. Desde entonces se ha convertido en una herramienta extensamente empleada para predecir el éxito del procedimiento y ha sido validado recientemente en cohortes contemporáneas. Recientemente, el estudio RECHARGE encontró en 880 procedimientos que las variables asociadas a una ATC-OTC no exitosa en pacientes abordados bajo el algoritmo híbrido son: bypass previo al vaso tratado, blunt stump, calcificación severa, tortuosidad que genere angulaciones superiores a 45 grados, longitud de la lesión $>20 \mathrm{~mm}$ y enfermedad significativa en el landing zone, recibiendo cada variable 1 punto. Se obtuvo un AUC de 0,78 en la cohorte de derivación y de 0,71 en la cohorte de validación con una calibración adecuada en ambas cohortes. Cuando se comparó este sistema con el J-CTO score y el PROGRESS score, la capacidad predictiva del modelo generado en el estudio RECHARGE fue superior que la de estos últimos dos.

De esta forma se pretende optimizar los tiempos de procedimiento con una alta tasa de éxito. Es importante que cada grupo adopte su propia "mirada", adoptando una sistemática de trabajo que sea capaz de acomodarse a los desafíos particulares que plantee cada lesión.

\section{Importancia del algoritmo híbrido en la actualidad}

La introducción del abordaje híbrido se asoció a una mayor tasa de éxito técnico definida como lesión residual menor a 30\% con flujo TIMI 3 y del procedimiento (éxito técnico sin desarrollo de complicaciones cardiovasculares mayores $)^{54}$. Mientras que previamente se reportaban tasas de éxito de alrededor del $76 \%$, el desarrollo de este algoritmo asociado a las mejoras en los dispositivos llevó a una tasa 


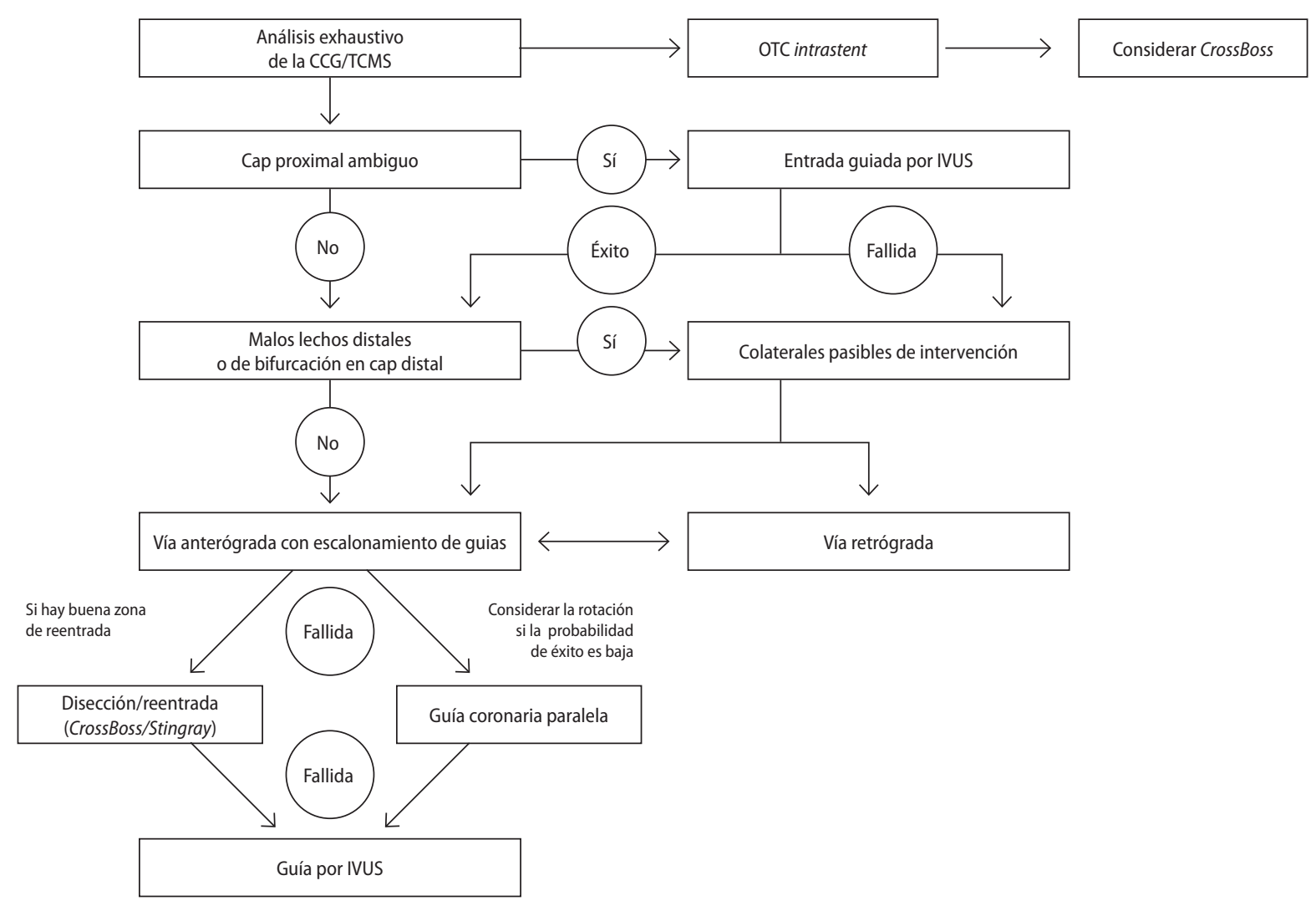

Figura 2. Algoritmo Asia-Pacífico.

de éxito sustancialmente superior (91\%, aproximadamente). En este trabajo el éxito del cruce se alcanzó por vía anterógrada en el $40 \%$, por vía retrógrada en el $32 \%$ y por la técnica disección y reentrada en el $28 \%$. Esto pone de manifiesto la necesidad de manejar adecuadamente las diferentes técnicas de cruce. Datos más recientes reportados por el estudio RECHARGE han evidenciado que con la incorporación de técnicas de disección y reentrada por vía anterógrada con el empleo del sistema CrossBoss-Stingray, el cruce exitoso por la vía anterógrada alcanzó el $74 \% \%^{55}$. Otro concepto de importancia es decidir cuándo dar por finalizado el procedimiento. El grupo de intervencionistas en OTC Asia-Pacífico ha establecido las siguientes recomendaciones ${ }^{56}$ :

- Más de 3 horas de procedimiento.

- $\quad>3,7$ x eGFR de ml de contraste.

- Kerma en aire >5 Gy.

\section{ALGORITMO ASIA-PACÍFICO}

Otro algoritmo muy completo, que representa la visión de la escuela japonesa es el algoritmo Asia-Pacífico. Como características distintivas, en él se utiliza ampliamente la angiotomografía coronaria multicorte para la evaluación de la anatomía coronaria, como también el ultrasonido intravascular para definir un cap ambiguo y para guiar la re-entrada de la guía coronaria a luz verdadera cuando se emplean técnicas de disección. Por otra parte, enfatizan en el estudio profundo de las colaterales y limitan la utilización de técnicas de reentrada en favor de técnicas de cruce por luz real ${ }^{56}$.

\section{MÉTODOS DE IMÁGENES COMPLEMENTARIOS PARA VALORAR LAS OCLUSIONES TOTALES CRÓNICAS}

\section{Rol del ultrasonido intracoronario en la angioplastia de las OTC}

El ultrasonido intracoronario (IVUS), nos proporciona imágenes del corte transversal de las arterias coronarias, que nos permiten conocer información anatómica valiosa respecto a la luz, la pared, así como la composición y la distribución de las placas ateromatosas en las arterias coronarias. Además, el IVUS puede ser muy útil para clarificar estructuras anatómicas, que no pueden ser identificadas satisfactoriamente por la angiografía, como puede ser el cap proximal de una OTC.

Es también particularmente útil en procedimientos complejos de intervenciones de OTC cuando por ejemplo debemos identificar el trayecto o la posición de una cuerda coronaria durante un acceso retrógrado. Asimismo, luego de la recanalización de una OTC, la dimensión real del vaso distal es frecuentemente subestimada y la elección del tamaño del/los stents basada solamente en la angiografía puede derivar en subexpansión del mismo. En estos casos, 
el implante de stents guiado por IVUS puede resultar en mejores resultados a largo plazo.

A pesar de lo mencionado, y que las más recientes guías europeas recomiendan el uso de IVUS para optimizar los resultados del implante de stents (Clase IIa, nivel de evidencia B), el IVUS es utilizado en una minoría de casos de intervenciones en OTC. En el Registro Europeo de OTC de 2011 ( $\mathrm{n}=1914)$, el IVUS fue utilizado sólo en $2,9 \%$ de casos $^{57}$.

\section{Evidencia clínica para el uso de IVUS en OTC}

El IVUS ofrece el potencial para superar desafíos técnicos en el tratamiento percutáneo de las OTC. Diferentes reportes de casos describen el avance del catéter de IVUS a una rama secundaria para identificar el punto de entrada de la obstrucción en la rama principal y también el avance del catéter de IVUS al falso lumen para permitir la visualización de la luz verdadera ${ }^{58-60}$.

Una serie pequeña de casos $(\mathrm{n}=31)$ mostró que la técnica de guiar la cuerda coronaria en casos de OTC sin muñón proximal es útil y segura, mientras que otra serie pequeña de casos, mostró elevado éxito del uso de IVUS para guiar la técnica de CART ${ }^{61}$.

Sin embargo, aún existen interrogantes y preocupación respecto a complicaciones potenciales y mayor duración del procedimiento de ATC en OTC con el uso de IVUS y los datos respecto a la relación entre el uso de IVUS para guiar la recanalización exitosa de una OTC y eventos clínicos posimplante de stent son escasos.

Típicamente, los estudios clínicos no han incluido el número de pacientes adecuado, para determinar el impacto del uso de IVUS sobre eventos clínicos en el seguimiento.

En el estudio AVIO (Angiography versus IVUS optimization), que incluyó pacientes con OTC, la utilización de IVUS no demostró ventajas en términos de reducción de eventos adversos cardíacos mayores a 24 meses de seguimiento ${ }^{62}$.

En 2014, en un análisis del Registro Coreano de OTC, la angioplastia fue guiada por IVUS en 206 pacientes, que fueron comparados con un grupo control de 201 pacientes en los que se guió la intervención con angiografía $^{63}$. A dos años el grupo guiado por IVUS mostró una trombosis del stent significativamente menor que el grupo de intervención guiada por angiografía ( 0 vs. 3\%; $\mathrm{p}=0,014)$ y una menor incidencia de infarto de miocardio ( 1 vs. $4 \% ; \mathrm{p}=0,058)$ que no alcanzó significancia estadística, probablemente debido a un error $\beta$.

En un estudio del año 2015, 230 pacientes portadores de OTC fueron randomizados luego de la recanalización exitosa, a colocación de stent guiada por IVUS o angiografía $^{64}$. En el grupo guiado por IVUS, la pérdida luminal tardía y la estenosis angiográfica fue significativamente menor que en el grupo guiado por angiografía. Sin embargo, esto no se acompañó de una menor incidencia de eventos cardíacos adversos mayores, probablemente debido a un tamaño de la muestra insuficiente.
En otro estudio de diseño similar al mencionado anteriormente, pero con mayor poder estadístico, 402 pacientes con OTC fueron aleatorizados a intervención guiada por IVUS $(n=201)$ o intervención guiada por angiografía $(\mathrm{n}=201)$. A 12 meses de seguimiento la tasa de eventos cardíacos adversos serios fue significativamente menor en el grupo guiado por IVUS que en el grupo guiado por angiografía $(2,6 \text { vs. } 7,1 \% ; \mathrm{p}=0,035)^{65}$.

Recientemente un trabajo publicado por Song y cols. evaluaron por IVUS el trayecto de la cuerda en 219 pacientes en quienes se pudo cruzar exitosamente una $\mathrm{OTC}^{66}$. Como resultados relevantes el IVUS detectó que en el $52,1 \%$ de los casos la cuerda tuvo un trayecto subintimal $(86,7 \%$ de las técnicas de disección y reentrada y en el 27,9\% de la técnica de escalonamiento de guías coronarias). Particularmente el grupo de trayecto subintimal se evidenció una mayor tasa del de eventos combinados de muerte, infarto y revascularización de la lesión culpable $(1,9$ vs. $7,9 \% ; \mathrm{p}=0,04)$, mayor tasa de contraste retenido $o$ extravasado $(3,8$ vs. $14 \%$; $\mathrm{p}=0,01)$ y una tendencia con significancia marginal a perforación clínicamente significativa ( $1 \%$ vs $6.1 \%, p$ p 0.07$)$. Estos resultados refuerzan la utilización del IVUS para efectuar un cruce endoluminal de la OTC en caso de ser posible. Merece la pena destacar que en este trabajo no se utilizó para disección y reentrada los sistemas CrossBoss y Stingray.

\section{Uso práctico del IVUS en las intervenciones en OTC}

Podríamos resumir que el uso de IVUS es beneficioso en las siguientes situaciones:

- En acceso anterógrado: Para ayudar en la identificación del cabo proximal de la OTC en situaciones angiográficas ambiguas y para guiar la cuerda coronaria hacia la luz verdadera, desde una posición subintimal.

- En acceso retrógrado: Para facilitar procedimientos de CART reverso, para prevenir el pasaje subintimal de una cuerda retrógrada en un vaso crítico, por ejemplo tronco de coronaria izquierda.

- Luego de la recanalización exitosa de la OTC: Para optimizar la expansión del stent, para clarificar la extensión de segmentos coronarios a cubrir con stent en caso de arterias difusamente enfermas.

\section{ROL DETOMOGRAFÍA CORONARIA MULTICORTE EN LA ANGIOPLASTIA DE LAS OTC}

La tomografía computada multislice coronaria (TCMS) es un método que permite la evaluación no invasiva de las arterias coronarias, visualizando el trayecto del vaso, la longitud de la oclusión y las características morfológicas de la placa en el sitio de oclusión. La TCMS cuenta con múltiples herramientas de reconstrucción como el análisis multiplanar curvo, el análisis longitudinal del vaso y la reconstrucción tridimensional.

Una oclusión total se define tomográficamente como la ausencia completa de contraste luminal. 
Existen diferentes variables tomografías que permiten predecir el éxito de la revascularización percutánea de las OTC, entre ellas las que han demostrado mayor poder son: - Longitud de la OTC.

- Composición de la placa (características morfológicas): - Placablanda

- Placa mixta con calcificación protruyente (ocupación por la placa cálcica, de la luz coronaria $>50 \%$.

- Placa mixta con calcificación no protruyente (ocupación por la placa cálcica de la luz coronaria $<50 \%)$.

- Placa cálcica pura.

- Oclusión abrupta o progresiva de la luz arterial al inicio y final de la lesión (blunt stop vs tapered stop).

En cuanto a la longitud, lesiones $<15 \mathrm{~mm}$ medidas por TCMS han demostrado mayor tasa de éxitos así como la composición predominantemente blanda o placas mixtas sin presencia de calcio protruyente y las lesiones tipo tapered.

Otras variables tomográficas con potencial utilidad en el planeamiento de la revascularización es la visualización de ramas y sus ángulos de emergencia, calibre de vasos, presencia y composición de otras lesiones obstructivas significativas y la presencia de circulación colateral.

De esta manera la TCMS constituye un método sumamente útil a la hora de valorar las posibilidades de éxito y planeamiento en el tratamiento percutáneo de las OTC, principalmente a través de la determinación de la longitud de las lesiones y la composición de las placas responsables de las OTC, con excelente repetitividad dada por la mínima variabilidad intra- e interobservador registrada.

\section{ABORDAJE ANTERÓGRADO CON ESCALAMIENTO DE GUÍAS CORONARIAS}

El abordaje anterógrado representa la estrategia más frecuente y la selección de la cuerda guía es una decisión basada entre las características de las mismas, herramientas de soporte y la anatomía arterial.

Actualmente la mayoría de los casos OTC se realizan con éxito por abordaje anterógrado con escalamiento de guías coronarias $^{57}$. El concepto fundamental en esta técnica se basa en el avance de la cuerda con menor fuerza sobre el microcatéter y progresando a cuerdas de mayor gramaje con el fin de conectar el cap proximal y dista ${ }^{41}$.

Existen diferentes factores anatómicos que favorecen el abordaje anterógrado. Estos puntos son los descriptos en el J-CTO score (longitud de la oclusión, características del cap proximal, tortuosidad $>45^{\circ}$, calcificación, intentos de recanalización).

\section{Longitud de la lesión}

La longitud de la OTC es un predictor independiente de fracaso y aumenta la tasa de cruce subintimal de la guía ${ }^{36}$. Con lesiones mayores de $20 \mathrm{~mm}$ se podría implementar técnicas de disección o abordaje retrógrado.

\section{Anatomía del CAP proximal}

La determinación del cap proximal es fundamental para el éxito del procedimiento. Es la zona con mayor contenido de tejido fibroso y calcificación. Un cap proximal romo o ambiguo es más probable de ser resistente a la penetración que el cónico, con una mayor necesidad implícita para guías coronarias con una alta fuerza de penetración.

\section{Tortuosidad}

La tortuosidad dentro del segmento de OTC se asocia tanto con un mayor riesgo de paso de la guía subintimal como con perforación del vaso, sobre todo cuando se asocia a calcificación ${ }^{42}$. En muchos de estos casos se debe rotar de estrategia ${ }^{67,68}$.

\section{Calcificación}

La presencia de calcio indica una mayor necesidad de guías de penetración. Cuando el calcio es muy severo y se asocia a un cap proximal romo, puede que no sea posible penetrar con la actual generación de cuerdas.

\section{Intentos de recanalización}

Existen diferentes factores aditivos relacionados con el procedimiento inicial, que pueden crear dificultades subsiguientes, tales como la creación de planos de disección.

\section{Otros factores}

Grado de la enfermedad en la "zona del cap distal". Una zona distal del cap altamente enferma puede afectar negativamente a la posibilidad de cualquier estrategia anterógrada y puede favorecer, en caso extremo, un procedimiento retrógrado primario. Asimismo, puede afectar el reingreso en el cap distal.

\section{Selección de la guía coronaria}

Una selección de cuatro cuerdas dedicadas para OTC cubrirá la mayor parte de las anatomías. Se pueden dividir en:

\section{Guías revestidas de polímero}

La cuerda recubierta de polímero le permite negociar entornos de placa densa. El bajo peso en gramos, combinado al recubrimiento de polímero distal le confiere menor tasa de perforación o disección inadvertida, por lo que son a menudo elegidas como la guía de primera de elección ${ }^{57}$. Ejemplos de ellas son la Fielder XT, Fielder XT-A o Fielder XT-R.

\section{Guías de gramaje medio}

Estos tipos dependen de la punta, recubrimiento y la capacidad de transmisión. Generalmente permiten atravesar el cap, cuando las cuerdas de polímero de bajo gramaje no han logrado progresar. Ejemplo Pilot 150 о 200.

\section{Guías de alto gramaje}

Estas cuerdas están diseñadas para penetrar una placa 
oclusiva, en particular cuando se encuentran severamente calcificadas. Ejemplos: Confianza Pro 12, o Progress.

\section{Guías de gramaje medio, con torque rígido}

Esta es la familia de guías Gaia. Éstas, tienen una punta cónica que a su vez las hace extremadamente rígidas y resistente con el torque, pero su punta seguirá siendo deflectiva cuando se empuja hacia adelante. Las rotaciones deben ser mínimas, limitadas a $90^{\circ}$ en cada dirección. Se establece una combinación de empuje y giro. Se debe tener muy en cuenta por ejemplo cuando se utilizan en OTC con cap ambiguo.

\section{Selección de guías: principios y fundamentos}

Se sugiere elegir las guías sobre la base de la función y el curso de la OTC que se está atravesando, y dependen de los siguientes factores:

1. Guias recubiertas de polímero: estas se caracterizan por ser más lúbricas, pero con menor sensación táctil. Asimismo, presentan una menor distinción de paso entre luminal y subintimal, debiéndose aplicar una rotación suave. Se recomiendan en OTC tortuosas.

2. Fuerza depenetración: se relaciona íntimamente con el gramaje. Pudiendo aumentar al avanzar sobre un microcatéter o balón.

3. Sensación táctil: La sensación táctil de un alambre será mejorada si la punta está sin recubrimiento. Esto significa que la mayoría de la resistencia está en ese segmento.

\section{Cruce del cap proximal}

La presencia de un cap proximal cónico, puede ser a menudo cruzada con una combinación de una cuerda de bajo gramaje con polímero y una de medio gramaje.

La presencia de calcificación severa requerirá, guías de alto gramaje, que deben ser usadas selectivamente para la penetración, sólo cuando el rumbo del vaso es claro. La inyección de contraste a través del microcatéter es raramente útil (en ausencia de colaterales ipsilateral) y puede ser perjudicial ya que puede causar una disección hidráulica inducida por contraste, o mediante la ampliación de los planos de disección inadvertidas.

\section{Cruce del cap distal}

El avance fallido de balones a través de la OTC provoca un prolapso hacia atrás del catéter guía. Para ello se pueden tomar en cuenta las siguientes recomendaciones:

- Balón de anclaje: Una cuerda se coloca en una rama lateral proximal con un balón de tamaño 1:1, el cual se infla a bajas atmósferas. Esto permite aumentar la fuerza para atravesar la oclusión.

- Guía de extensión y soporte: el GuideLiner o GuideZilla hasta el punto de la oclusión aumenta en gran medida la cantidad de fuerza coaxial que puede ser entregada ${ }^{69}$.

- Soporte superior del microcatéter: El Tornus es un microcatéter que permite al rotar aumentar las oportu- nidades de cruce, al traducirse en avance del mismo ${ }^{70}$. Las mismas se realizan en sentido contrario, con un máximo de 20 giros, con el fin de no alterar la arquitectura del catéter.

\section{TÉCNICA DE DISECCIÓN ANTERÓGRADA Y REENTRADA}

\section{Indicaciones}

Si bien la mayoría de las OTC serán abordadas por la técnica anterógrada con escalamiento de guías coronarias (ver apartado anterior), el éxito de esta técnica es inversamente proporcional a la complejidad de las lesiones $\mathbf{5 7 , 3 6 .}$ Además, si bien la técnica de recanalización retrógrada permite la reapertura del vaso a través de su lumen verdadero, muchas veces se ve limitada por la falta de colaterales apropiadas. Esto último no es menor, dado que aproximadamente el 36,5\% de las OTC estudiadas no presentan colaterales apropiadas ${ }^{49}$.

La técnica de disección anterógrada y reentrada (DAR) es una alternativa que ha demostrado una significativa tasa de éxito en casos en los que las otras estrategias han falla$\mathrm{do}^{71}$. Incluso en la actualidad se ha empleado como la primera línea de abordaje en el 30\% de los casos ${ }^{55}$.

Como regla general, todas las características que constituyen desventajas para las técnicas de recanalización endoluminal se convierten en potenciales indicaciones para la técnica de DAR. Los casos ideales son aquellos con un cap proximal definido, trayectos ocluidos largos (>20 mm.), con vaso distal de buena calidad y que tenga adecuada visualización para permitir la reentrada. El vaso distal a la oclusión (en la literatura anglosajona landing zone) idealmente deberá presentar un buen calibre y ausencia de calcificación severa. Un factor determinante es que la landing zone no se encuentre en relación a una ramificación importante del vaso por el riesgo aumentado de oclusión de la misma ${ }^{72}$. Otras situaciones en las que las técnicas de DAR tienen indicación son la ausencia de circulación colateral para el abordaje retrógrado como bail out cuando se entra por disección subintimal durante el intento de cruzar por vía anterógrada ${ }^{73}$.

\section{TÉCNICA STAR Y SUS VARIANTES}

La técnica de DAR ha experimentado significativos progresos con el tiempo. Inicialmente se empleaba la técnica conocida como STAR ${ }^{74}$. La misma consiste en doblar la parte distal de una guía 0,014" hidrofílica creando un asa que es empujada hacia el lumen distal, con el apoyo de un microcatéter. De este modo, el asa progresará hacia el punto de menor resistencia en el espacio subintimal. La reconexión entre el lumen verdadero proximal y el distal se logrará en el punto de reentrada, típicamente en los sitios de bifurcación. Con esta técnica el punto de reentrada es impredecible, pudiendo generar trayectos subintimales muy largos, con la consiguiente oclusión de ramas laterales. Asimismo, en otras ocasiones la reentrada no puede pro- 
ducirse en el lumen del vaso principal y hacerlo en el lumen de un vaso pequeño, con un outflow poco significativo y una landing zone inadecuada para un stent $t^{74}$.

Para corregir esto, se implementó la técnica STAR con contraste, que consiste en inyectar material de contraste a través del microcatéter en el espacio subintimal para lograr una "disección hidráulica" y, a su vez, una mejor visualización que permita obtener una reentrada más controlada ${ }^{75}$. Otra modificación es la técnica LAST (Limited Antegrade Subintimal Tracking) en la que una vez que la guía y el microcatéter alcanzan el segmento distal de la lesión por el espacio subintimal, se utiliza una guía coronaria con fuerza de penetración, a la cual se le otorga una curvatura generosa ${ }^{76}$.

Desafortunadamente, todas estas técnicas han resultado ser muy desafiantes e impredecibles. El fracaso generalmente es debido a la imposibilidad de reentrada al lumen verdadero distal, única vía para permitir la reconstrucción del vaso. La disrupción tisular generada por el avance de la guía con asa más la manipulación de la guía con punta rígida en sus intentos de perforación, terminan produciendo un gran hematoma subintimal, el cual comprime a la luz verdadera del vaso distal a la oclusión, disminuyendo las chances de reentrar al mismo. Las inyecciones de contraste a través del microcatéter pueden generar aún más daño (contrastoma) y empeorar la situación ${ }^{76}$.

Debido al poco control, tanto del avance de las guías como al proceso de reentrada, estas técnicas se asocian a una mayor tasa de perforaciones. A su vez, otras consecuencias técnicas indeseables, como la oclusión de ramas adyacentes y la necesidad de cubrir mayores longitudes con stents tienen su impacto clínico. En las series publicadas con seguimiento a largo plazo, las técnicas de DAR se asocian a una mayor tasa de $\operatorname{TLR}^{77,78}$.

\section{USO DEL CATÉTER CROSSBOSS Y EL BALÓN STINGRAY}

El catéter CrossBoss y el balón Stingray (Boston Scientific) constituyen un sistema creado para permitir una disección anterógrada controlada que facilite la reentrada en un sitio deseado y seguro ${ }^{71}$.

El CrossBoss es un microcatéter de punta roma de $1 \mathrm{~mm}$ con cobertura hidrofílica y un cuerpo reforzado con un alambre dispuesto en forma espiralada. Es compatible con catéter guía $6 \mathrm{~F}$ y admite guías 0,014 ". Para avanzarlo se lo rota rápidamente a través de un torque proximal sin que la guía 0,014" sobrepase el extremo distal del mismo. El CrossBoss puede abrir su camino a través del lumen verdadero o el espacio subintimal. En ocasiones el mismo microcatéter reingresa al lumen distal.

En caso de imposibilidad de progresar el microcatéter, se puede avanzar una guía con asa a través del espacio subintimal, durante un corto trayecto. El avance de guía con asa genera mayor disrupción subintimal y hematoma con colapso de la luz verdadera por lo que se recomienda que el tramo final de la disección se realice con el catéter Cross-
Boss el cual genera un trayecto con menos disrupción y hematoma y por ende menor compresión en el sitio de reentrada.

Una vez elegido el sitio de reentrada mediante angiografías con doble inyección puede combinarse al catéter CrossBoss la utilización del balón Stingray. Se trata de un balón plano de $10 \mathrm{~mm}$ de longitud por 2,5 $\mathrm{mm}$ de ancho. El mismo se infla a baja presión (4 atmósferas) en el sitio elegido para la reentrada. La conformación plana del balón permite que una vez insuflado, abrace al lumen verdadero. El balón cuenta con dos orificios laterales orientados cada uno a las caras opuestas del mismo. Para la reentrada se recomienda el uso de la guía hidrofílica Stingray, con punta cónica de 0.09 ", aunque admite el tipo de guía de 0,014" a elección del operador.

La elección del sitio de reentrada y la elección de la óptima proyección de trabajo serán cruciales para el éxito del procedimiento. En primer lugar, se debe lograr una orientación que genere el menor acortamiento longitudinal del balón. El segundo punto será lograr, bajo control radioscópico, que las dos aletas del balón queden orientadas en forma paralela al haz de rayos. En otras palabras, debe verse solo una línea. Finalmente, el lumen distal debe ser perfectamente identificado a través de llenado retrógrado. De este modo uno de los dos orificios del balón siempre estarán orientados hacia la luz verdadera, siendo en esta dirección hacia donde debe dirigirse la guía de reentrada.

Una vez conseguida la reentrada, el balón Stingray puede removerse e intercambiarse por un microcatéter utilizando la técnica de Trapping insuflando un balón dentro del catéter guía. Una vez posicionado el microcatéter dentro de la luz verdadera puede intercambiarse la guía por una menos agresiva y controlable para terminar la angioplastia. La gran ventaja de esta técnica es que permite elegir el sitio de reentrada, evitando la oclusión de ramas laterales. Cuando el vaso es reconstruido con stents, la permeabilidad de las mismas estará garantizada.

A su vez, los resultados a largo plazo de esta técnica son más comparables a los de las técnicas de recanalización endoluminal.

Los resultados del estudio RECHARGE publicados recientemente demostraron que el empleo de la técnica DAR controlada con CrossBoss y Stingray es segura y eficaz. La misma fue empleada en el 23\% de los casos, particularmente en lesiones complejas (> J-CTO score, OTC asociada a reestenosis intrastent, lesiones $>20 \mathrm{~mm}$, blunt stump, cap ambiguo, tortuosidad severa). El éxito de la DAR cuando fue la estrategia inicial fue del $67 \%$ mientras que cuando se utilizó como bail out fue del 63\%. Finalmente, comparado con la vía anterógrada con escalonamiento de guías coronarias, la técnica DAR requirió de $>$ tiempo y dosis de fluoroscopía, > dosis de contraste y > $\mathrm{n}^{\circ}$ de guías coronarias ${ }^{55}$.

Recientemente, Wilson y cols. publicaron una cohorte de 969 pacientes abordados mediante el algoritmo híbrido y que en el $47 \%$ de los casos se empleó alguna técnica de disección y reentrada (anterógrada o retrógrada). La preva- 
lencia de muerte, infarto no fatal y revascularización no planeada al año de la intervención fue similar en ambos grupos, con una mejoría similar de la sintomatología ${ }^{79}$.

\section{DISECCIÓN SUBINTIMAL ANTERÓGRADA Y RETRÓGRADA CONTROLADAS Y SIMULTÁNEAS}

En algunos casos, podría darse la combinación de accesos subintimales tanto anterógrados como retrógrados. En estos casos, en el punto de encuentro suele insuflarse un balón pequeño $(1,5 \mathrm{~mm})$ en el espacio subintimal retrógrado para facilitar la perforación con la guía anterógrada y su acceso al lumen verdadero. Esta técnica es conocida como CART (controlled antegrade and retrograde subintimal tracking $)^{11}$.

Una variante a esta técnica, el CART inverso, consiste en la insuflación del balón en el espacio subintimal anterógrado, facilitando el pasaje de microcatéter y guía desde el espacio retrógrado.

\section{CONSIDERACIONES TÉCNICAS ESPECIALES}

Catéter guía y vía de abordaje: si bien está descripta la factibilidad y el éxito de las intervenciones en OTC por vía radial, cuando se planea la utilización de una técnica de DAR o bien existe la posibilidad de utilizarla como bail out, se recomienda la utilización de catéteres guía $8 \mathrm{~F}$, por tanto la vía femoral con introductor largo de no menos de $45 \mathrm{~cm}$ serán la elección.

IVUS: el uso de ultrasonido intravascular no solo será de utilidad para la selección de la longitud y diámetros de los stents a utilizar para la reconstrucción del vaso sino que muchas veces resultará crucial utilizándolo en "tiempo real" para la correcta definición de un sitio de oclusión proximal ambiguo. Para esta última situación, un catéter guía $8 \mathrm{~F}$ permitirá el avance simultáneo de la sonda de ultrasonido y un microcatéter.

\section{ABORDAJE RETRÓGRADO}

Desde el primer reporte de abordaje retrógrado para el tratamiento de OTC, la recanalización a través de colaterales continúa siendo un desafío para la cardiología intervencionista $^{80}$. Su incorporación a la práctica diaria trajo consigo un aumento en la tasa de éxito en el tratamiento de lesiones complejas, algunas de ellas no pasibles de abordaje anterógrado $^{81,82}$.

Como se describe más adelante, este tipo de abordaje requiere no solo de las destrezas para el tratamiento de las OTC por vía anterógrada, sino también del conocimiento de diversos materiales y técnicas que raramente se utilizan en la práctica diaria. Es por esto que se recomienda que la estrategia retrógrada sea utilizada por grupos dedicados al tratamiento de OTC, con un caudal de 125 casos/año (20 por vía retrógrada $)^{7,82}$. Thompson y cols. demostraron di- ferencias significativas en la tasa de éxito, tiempo de fluoroscopia y volumen de contraste utilizado entre grupos entrenados y aquellos no preparados para este abordaje ${ }^{7}$. Es así que en grupos entrenados, la tasa de éxitos se sitúa alrededor de $80 \%$, siendo menor cuando es utilizada como bail-out ante un fracaso de la técnica anterógrada ${ }^{83,84}$.

\section{ELECCIÓN DE LA ESTRATEGIA TERAPÉUTICA}

Como se ha comentado, el algoritmo hibrido es de elección para abordar la estrategia de recanalización de OTC $^{13}$. Este comienza con inyección doble para evaluar parámetros angiográficos que permitan seleccionar el abordaje inicial. La estrategia inicial puede cambiar de acuerdo a la evolución del caso y al criterio del operador. La doble inyección nos provee de información crítica: no solo la longitud de la lesión, cap proximal, calcificación y tortuosidad (parámetros utilizados en el J-CTO score), sino también el tamaño del vaso distal, el monto de miocardio amenazado y la presencia y características de las colaterales ${ }^{85}$. Sobre este punto, vale diferenciar las colaterales epicárdicas de las septales: estas últimas son más seguras por lo que deberían ser la primera elección siempre que se pueda. Las colaterales epicárdicas en general son más largas y tortuosas, por lo que su utilización debería reservarse en casos donde no se pueda avanzar por vasos septales.

Respecto al diámetro del vaso distal a la oclusión, vale recordar que puede estar disminuido debido a un estado de hipoflujo y es común que crezca luego de la angioplastia, o en el seguimiento a mediano plazo. Por esto es muy importante tener en cuenta el monto de miocardio amenazado a la hora de encarar el tratamiento de una OTC ${ }^{13}$.

\section{PREPARACIÓN PARA EL ABORDAJE RETRÓGRADO}

Se deben tener en cuenta algunos puntos antes de comenzar un abordaje retrógrado. En primer lugar debemos considerar la elección de los catéteres guías; es preferible utilizar catéteres guía de $7 \mathrm{~F}$ en el lado anterógrado, dado que es conveniente tener en ese lado el mejor lumen posible, no solo para facilitar el uso de materiales tales como IVUS o extensores de catéteres sino también porque teniendo un catéter de mayor lumen será mucho más fácil ingresar al guía con la cuerda retrógrada. Por otro lado, el catéter guía a utilizar en el lado retrógrado puede ser de menor diámetro, ya sea 5 o $6 \mathrm{~F}$. El uso de estos catéteres facilita la realización de maniobras de intubación profunda, si fuera necesario para obtener un mejor apoyo.

En el apartado de abordaje híbrido se describieron con detalle los conductos que pueden emplearse para el abordaje retrógrado:

- Vasos colaterales. Estos pueden ser septales, epicárdicos o intramiocárdicos.

- Puentes quirúrgicos. Estos pueden ser arteriales o venosos. 


\section{Vasos colaterales}

Dentro de los vasos colaterales, siempre es preferible utilizar los colaterales septales, ya que son mucho más seguros, dado que ante una ruptura o perforación, usualmente generan hematomas septales que se autolimitan o bien drenan hacia la cavidad ventricular. En caso de ruptura y hematoma de ramo septal debemos seguir de cerca a estos pacientes dado que, aunque muy rara vez, pueden provocar trastornos de la conducción intraventricular o complicaciones mecánicas de tipo comunicación interventricular u obstrucción transitoria del tracto de salida del ventrículo izquierdo.

Por otra parte las colaterales epicárdicas suelen ser vasos más frágiles y por su localización, en caso de ruptura o perforación, drenan hacia la cavidad pericárdica con el consiguiente riesgo de taponamiento cardíaco. Este tipo de colaterales deberían reservarse para los pacientes con cirugía cardíaca previa, que no conllevan riesgo de taponamiento y siempre y cuando se trate de colaterales de buen tamaño y poco tortuosas. Por último, las colaterales intramiocárdicas suelen ser de difícil acceso, y el avance de los microcatéteres suele resultar dificultoso debido a la compresión miocárdica, esto las transforma en canales colaterales de riesgo para ser abordados, dado que en caso de ruptura, suelen disecar el miocardio circundante y conducen al taponamiento cardíaco; al igual que las colaterales epicárdicas, no debieran ser utilizadas en pacientes con pericardio intacto.

\section{Puentes venosos}

Los puentes quirúrgicos ya sean venosos o arteriales pueden ser utilizados como vía de abordaje retrógrado en diversas circunstancias, como por ejemplo mejorar el flujo anterógrado a un ramo diagonal, abriendo una DA proximal ocluida a través de un puente mamario permeable, o utilizar el puente mamario como vía de ingreso a las septales para abrir una oclusión de coronaria derecha. También se puede utilizar como conducto retrógrado un puente venoso ya tratado con stents, el cual presentó reestenosis reiteradas, para abrir el vaso nativo ocluido, asumiendo una alta tasa de re-reestenosis del puente venoso. Al utilizar puentes quirúrgicos como conducto se debe prestar particular atención a: en el caso de puentes arteriales, no utilizarlos si presentan extremada tortuosidad, sobre todo si son la única fuente de flujo hacia el vaso tributario del puente; en el caso de los puentes mamarios, tener en cuenta que el ostium de la arteria mamaria interna es frágil y el riesgo de disección con el catéter guía no es menor, sobre todo si no se presta mucha atención en las maniobras de intubación. En lo que respecta a los puentes venosos, tener en cuenta que si se trata de puentes de larga data el riesgo de desprendimiento de material friable de la pared es alto, con el consiguiente desarrollo de fenómeno de no reflow; este riesgo es menor en los puentes tratados previamente con stents.

\section{Técnica recomendada para el abordaje retrógrado}

La técnica de abordaje retrógrado siempre involucra el uso de un microcatéter, avanzado sobre una cuerda de punta blanda, utilizada para posicionarlo en la colateral. Una vez que el microcatéter se encuentra en posición, es recomendable cambiar a una cuerda hidrofílica de punta blanda y con ella avanzar sobre la colateral, en algunas ocasiones puede ser útil avanzar el microcatéter dentro de la colateral, quitar la cuerda y realizar una inyección de contraste a través del microcatéter para visualizar mejor el trayecto. Una vez que se logra avanzar la cuerda hacia el lecho distal el vaso ocluido a través de la colateral se debe avanzar el microcatéter. Los microcatéteres mallados con capacidad de rotación como el Corsaire son preferibles, sobre todo cuando se utilizan colaterales septales, ya que actúan como dilatadores. Los microcatéteres de bajo perfil como el FineCross son preferibles cuando se abordan colaterales epicárdicas o puentes arteriales, ya que en estos casos no es necesario dilatar el conducto y por su bajo perfil es menos probable que provoquen alteración del flujo. El objetivo final de toda técnica retrógrada es llegar con la punta del microcatéter lo más cerca posible del cap distal de la oclusión. Una vez alcanzado este punto, se intercambiará la cuerda por una cuerda de mayor gramaje dedicada para OTC, y con esta se tratará de avanzar a través del cap distal, eligiendo alguna de las dos técnicas que se describen a continuación.

\section{Punción retrógrada de luz verdadera}

Es la técnica más utilizada cuando se trata de oclusiones cortas (menos de $20 \mathrm{~mm}$ ). El objetivo es tratar de penetrar el cap distal y avanzar hacia la luz verdadera proximal. Es recomendable utilizar un dispositivo del tipo Mother and Child o Guidezilla desde el lado anterógrado para así acortar la distancia a recorrer con la cuerda de alto gramaje. Una vez ganada la luz verdadera se ingresará con la cuerda en forma retrógrada dentro del catéter guía del lado anterógrado, para recuperarla desde este lado y continuar con la angioplastia de manera convencional.

\section{Disección re-entrada retrógrada}

En esta técnica la cuerda retrógrada es avanzada a través del espacio subintimal y por otro lado una cuerda anterógrada es avanzada también por el espacio subintimal, se procede luego a avanzar un balón por la cuerda anterógrada e insuflarlo para ampliar el espacio subintimal para luego intentar reentrar en este espacio con la cuerda retrógrada. Cuando se utiliza esta técnica, es de suma utilidad el ultrasonido endovascular, para ayudar a diferenciar entre luz verdadera y luz falsa.

\section{MATERIALES ¿QUÉ NO PUEDE FALTAR AL MOMENTO DE REALIZAR UNA ANGIOPLASTIA A OCLUSIONES TOTALES CRÓNICAS?}

La implementación de un programa de OTC requiere no solo el conocimiento detallado de la técnica, sino también 
estar familiarizados con el creciente espectro de materiales dedicados al tratamiento de estas lesiones. Contar con el material adecuado no solo significa una mayor eficacia, sino también una mayor eficiencia operativa.

\section{Acceso vascular}

Siguiendo las pautas del algoritmo híbrido, es fundamental realizar doble acceso con el fin de guiar el procedimiento por inyección desde el vaso dador de circulación colateral y para poder rotar la técnica de abordaje cuando sea necesario. En este sentido, los accesos empleados dependerán de las preferencias del operador y de cada caso en particular (por ejemplo, pacientes con enfermedad vascular periférica grave). Podrá optarse por acceso bifemoral, birradial o combinado.

El acceso bifemoral con introductores de $45 \mathrm{~cm}$ es el preferido por los expertos dado que brinda un soporte adecuado al catéter guía y mejora el torque del mismo al negociar la tortuosidad ilíaca. Además, acepta introductores y catéteres de mayor diámetro sin ninguna dificultad $(7 \mathrm{~F}, 8 \mathrm{~F})$, los cuales no son infrecuentes de utilizar en estas angioplastias. En Europa alrededor del 90\% de los operadores utiliza la vía femoral ${ }^{86}$.

Sin embargo, con la creciente experiencia y el mayor repertorio de materiales dedicados, el acceso radial está cobrando mayor relevancia. En la actualidad el acceso radial se ha empleado con la misma seguridad y eficacia que el femoral en la angioplastia a OTC incluso en lesiones con mayor complejidad medida por el score J-CTO ${ }^{87}$. No obstante, los pacientes del grupo femoral más frecuentemente recibieron un abordaje híbrido con doble inyección $(80 \%$ vs. $53 \% ; \mathrm{p}<0,0001)$. A pesar de esto, la tasa de éxito técnica y del procedimiento han sido altas y similares en ambos grupos. Si bien no se observaron diferencias significativas en cuanto a los desenlaces cardiovasculares, el grupo radial presentó mayor tiempo de procedimiento y mayor tiempo de fluoroscopia.

Datos publicados recientemente del registro RECHARGE evidenciaron que el uso de abordaje completamente por vía radial fue del $24 \%^{88}$. En este caso la complejidad de la lesión fue mayor en el grupo femoral aunque no se alcanzó significancia estadística. A su vez, las tasas de complicaciones vasculares mayores fueron similares entre ambos grupos y a diferencia del estudio descripto previamente, no se observaron diferencias significativas en cuanto a la dosis radiación aunque sí se observó una menor utilización de contraste por vía radial.

En caso de necesitar catéteres de gran diámetro $(8 \mathrm{~F})$, se han reportado cohortes con escaso número de pacientes en los que se utilizó exitosamente un catéter guía $8 \mathrm{~F}$ sin introductor por vía radial en forma exitosa y con un bajo número de complicaciones vasculares ${ }^{89}$.

La complejidad anatómica de la lesión también tendrá un rol predominante en la elección del tamaño del acceso. Recomendamos planificar la intervención inicialmente con accesos $\geq 7 \mathrm{~F}$ en el caso de lesiones complejas (tipo blunt stump $>20 \mathrm{~mm}$, calcificación severa, pla-

\begin{tabular}{|c|c|}
\hline $\begin{array}{l}\text { El acceso radial es importante } \\
\text { para el éxito de la angioplastia a } \\
\text { OTC }\end{array}$ & $\begin{array}{l}\text { El acceso femoral común es pre- } \\
\text { ferido durante una PCI de OTC }\end{array}$ \\
\hline $\begin{array}{l}\text { En pacientes con CRM previa cuan- } \\
\text { do la ATC-OTC requiere más de } \\
\text { dos accesos arteriales. } \\
\text { La necesidad de utilizar dispositivos } \\
\text { de soporte hemodinámicos por } \\
\text { acceso femoral. } \\
\text { Enfermedad arterial periférica con } \\
\text { oclusión de la aorta, iliaca o arte- } \\
\text { ria femoral común. } \\
\text { Pacientes con alto riesgo de compli- } \\
\text { caciones por acceso femoral (an- } \\
\text { ticoagulados). }\end{array}$ & $\begin{array}{l}\text { Arteria radial anatómicamente au- } \\
\text { sente (ausencia de pulso). } \\
\text { Arteria radial que no pueda acomo- } \\
\text { dar un introductor } 6 \mathrm{~F} \text { (o mayor). } \\
\text { Variación anatómica que impida el } \\
\text { cruce desde el acceso radial con } \\
\text { la guía o el catéter guía. } \\
\text { Severa tortuosidad de la arteria } \\
\text { subclavia o del tronco braquio- } \\
\text { cefálico } \\
\text { Pacientes que puedan necesitar un } \\
\text { acceso permanente para hemo- } \\
\text { diálisis. } \\
\text { Oclusión total crónica ostial o muy } \\
\text { proximal. }\end{array}$ \\
\hline
\end{tabular}

nificación de utilizar IVUS), mientras que las lesiones de menor complejidad (punta cónica, bajo contenido de calcio, $<20 \mathrm{~mm}$ ) podrían abordarse con catéteres $6 \mathrm{~F}$ en primera instancia.

\section{Catéteres guía}

Otro punto importante es la selección adecuada del catéter guía. Desde ya que la mejor opción dependerá de variables anatómicas, como así también de la preferencia del operador. Sin embargo, es innegociable el empleo de catéteres guía que brinden un soporte óptimo para llevar a cabo la intervención. La recomendación general es utilizar, de inicio, catéteres EBU o XB para la coronaria izquierda y catéter AL1 o AL2 para la coronaria derecha. Muchos operadores utilizan catéteres guía de $8 \mathrm{~F}$ cuando acceden por vía femoral. Es importante contar con catéteres con orificios laterales cuando se introducen frenchs elevados para evitar el dampeo de presiones, principalmente cuando utilizamos AL1 en la coronaria derecha. En caso de emplear catéteres que puedan resultar oclusivos para el ostium de la coronaria se podrían implementar orificios laterales al catéter.

Un aspecto de gran relevancia es evitar la pérdida de sangre durante el procedimiento utilizando llaves en $Y$ con válvulas hemostáticas (por ejemplo: Co-Pilot, Abbot Vascular o Guardian, Vascular Solution).

\section{Guías coronarias}

Es fundamental contar con un amplio repertorio de guías coronarias, como también conocer al detalle sus propiedades y potencial utilidad según el tipo de lesión a cruzar y el momento de la intervención. El proceso de cruzar una OTC podría dividirse en tres pasos grosso modo: a) penetrar el cap proximal, b) atravesar el cuerpo de la lesión y alcanzar el cap distal y c) penetrar el cap distal. Para ello se han diseñado numerosas cuerdas con las propiedades adecuadas para cumplir esta función.

Las mismas podrán clasificarse según presenten o no cubierta con polímeros, la forma y la rigidez de la punta.

Las cuerdas cubiertas de polímero o hidrofílicas ofrecen una gran maniobrabilidad así como la capacidad de localizar y navegar a través de microcanales. Sin embargo, 
hay que ser muy cuidadosos dado que pueden provocar disección subintimal, incluso perforación coronaria.

La cuerda Fielder XT (Asahi Intecc) presenta punta cónica $(0,010)$ y se trata de una cuerda suave con bajo gramaje $(1,2 \mathrm{~g})$. Es una de las cuerdas de primera elección para el abordaje anterógrado y también puede utilizarse para generar el asa con la cuerda para la disección y reentrada. Actualmente se presenta un diseño dedicado al abordaje anterógrado (Fielder XT-A) y otro para el retrógrado (Fielder $X T-R)$. Otras guías hidrofílicas de punta recta y con baja rigidez de la punta son la Fielder $F C$ (Asahi Intecc), Whisper LS, MS, ES (Abbot Vascular), Pilot 50 (Abbot Vascular) y Choice PT Floppy (Boston Scientific). Este grupo de cuerdas es especialmente útil para el abordaje retrógrado. El último grupo de cuerdas hidrofílicas también presenta punta recta pero la rigidez de la punta es mayor. Entre los referentes encontramos: Pilot 150 y Pilot 200 (Abbot Vascular). Estas son útiles para el cruce anterógrado de la placa, especialmente cuando se trata de un cap ambiguo y también podrán utilizarse para realizar el asa para la disección y reentrada. No están diseñadas para navegar las colaterales, dado que su punta rígida puede romper estos vasos.

Las cuerdas no cubiertas con polímero o no hidrofílicas son más controlables, brindan una mejor sensación táctil y es menos probable que provoquen disección. Como tienen mayor fuerza de penetración son una buena opción para cruzar un cap fibroso.

Las cuerdas de este grupo con punta recta son las SION (Asahi Intecc). La misma presenta toque 1:1, con excelente conservación de la curva en la punta. Son de primera elección para navegar a través de colaterales. Otro grupo de cuerdas son las de punta cónica y con poca fuerza: Cross-it 100XT (0.010in, Abbot Vascular) y la Runtrhough NS Tapered (0.008", Terumo). Otro grupo lo constituyen cuerdas de punta cónica con mayor rigidez: Confianza Pro9 y Pro12 (0.009", Asahi Intecc), PROGRESS 140 T, $200 T$ (Abbot Vascular). Estas cuerdas son adecuadas para la vía anterógrada cuando se conoce el trayecto del vaso. Otro grupo está representado por las Gaia1, Gaia2, Gaia3 (Asahi Intecc). Presentan torque 1:1 y presentan punta cónica que disminuye según la generación. Está diseñada para desviarse si encuentra una resistencia y es fácilmente dirigible dentro de la oclusión.

\section{Microcatéteres}

Son de gran utilidad para aumentar el soporte de la guía. En el abordaje anterógrado son imprescindibles, mientras que en el retrógrado son útiles para no perder la posición en caso de cambiar la guía coronaria y mejorar el soporte para avanzar la misma hacia el lecho distal de la arteria tratada. Si bien, en su lugar podrían utilizarse balones sobre cuerda, estos últimos son menos resistentes al kinking. Los microcatéteres más utilizados son:

- Corsair: presenta una gran respuesta al torque y luz interna cubierta de un polímero que facilita el avance de la guía. Su punta es cónica y suave y el extre- mo distal del catéter presenta una cubierta hidrofílica que aumenta la capacidad de cruce. Si bien puede avanzar rotándose en ambas direcciones, tiene más potencia cuando se rota en sentido antihorario, aunque debe evitarse la sobrerrotación dado que se puede deformar y la punta puede separarse del resto del catéter. Con el uso por tiempos prolongados puede sentirse una mayor resistencia en el paso de las guías coronarias, lo que se conoce como fatiga del Corsair y debe reemplazarse.

- Finecross: es el que presenta el menor perfil de cruce. De punta recta y suave, presenta una trenza de acero inoxidable que le brinda una gran capacidad de torque. Es especialmente útil para navegar vasos colaterales epicárdicos.

- Otros: Venture, MultiCross, Turnpike y Prodigy no están disponibles en Argentina.

\section{Extensores de catéteres}

Son de gran importancia para mejorar el soporte del catéter. Existen de dos tipos, unos rapid-exchange como el Guidezilla y el Guideliner. El primero solo se presenta en 6 F mientras que Guideliner viene en 4 tamaños: 5,5 F, 6 F, 7 F y 8 F. Otro sistema de extensión es el catéter Heartrail (Terumo) el cual es un segundo catéter guía $10 \mathrm{~cm}$ más largo que el primero. Si bien estos mejoran el soporte, hay algunos detalles que deberán ser tenidos en cuenta. Primero, disminuyen el diámetro del catéter en aproximadamente $1 \mathrm{~F}$. Segundo, se ha descripto deformación del sistema primario y la intubación dentro de la coronaria puede provocar disección.

\section{MANEJO DE LA RADIACIÓN YEL CONTRASTE EN ANGIOPLASTIA A OCLUSIONES TOTALES CRÓNICAS}

El abordaje exitoso de las OTC implica la aplicación de técnicas complejas que requieren el uso intensivo de recursos de radiación y medio de contraste, con el consiguiente riesgo potencial para el paciente y los operadores. Afortunadamente existe una serie de medidas cuya aplicación contribuye sustancialmente a reducir el uso y el efecto de estos recursos, limitando las complicaciones y permitiendo la extensión de la práctica a mayor número de pacientes.

\section{Radiación}

Existen dos formas en que los equipos modernos expresan la radiación que producen:

1. Producto dosis área (grays/área): expresa la dosis total de radiación entregada al paciente y se relaciona con los efectos estocásticos a largo plazo (malignidad/alteraciones genéticas).

2. Dosis de entrada o air kerma (grays): expresa la dosis entregada al paciente en el punto aproximado de ingreso del haz de rayos al paciente y se relacionan con los efectos determinísticos como lesiones de la piel. 
Ambas mediciones de dosis, al igual que el tiempo total de fluoroscopia están significativamente aumentados en los procedimientos de OTCen relación a otro tipo de procedimientos de cardiología intervencionista: 1,4 Gy para una sola lesión y 4,5 Gy para OTC ${ }^{90}$.

Es importante recordar que aunque rara vez hay toxicidad por radiación a dosis menores a 5 Gy, a partir de 2 Gy, 7 Gy y 12 Gy pueden producirse eritema transitorio, depilación permanente y necrosis dérmica, respectivamente, y que los efectos estocásticos a largo plazo están directamente relacionados con la dosis ${ }^{90}$.

Afortunadamente, es posible reducir significativamente la dosis de radiación para el paciente y los operadores siguiendo estrictamente las recomendaciones generales para los equipos emisores de rayos $\mathrm{X}^{91}$, adaptadas al tratamiento de OTC ${ }^{86,92}$, y referidas al equipamiento específico, la técnica radiológica y el monitoreo de dosis.

\section{Equipamiento}

- La disponibilidad de un angiógrafo actualizado con tubos de rayos $\mathrm{X}$ de alto rendimiento, fluoroscopia pulsada y filtro del haz de rayos (lo cual reduce la dosis de entrada al paciente y la radiación dispersa al operador) debería ser mandatoria ${ }^{86}$.

- La instalación y utilización sistemática de blindaje entre el tubo de rayos $\mathrm{X}$, el paciente y el/los operadores: cortinas plomadas laterales inferiores y pantalla plomada suspendida, reduce la radiación dispersa o secundaria entre 4 y 10 veces.

- Utilización sistemática de prendas plomadas: gafas, delantal etc.

\section{Técnica radiológica}

- Utilización de baja dosis de fluoroscopia como default: como ejemplo, $100 \mathrm{kv}$ cuando el preestablecido es $125 \mathrm{kv}$. Aunque esto puede aumentar el ruido de la imagen, si se mantiene un nivel de contraste adecuado puede ser suficiente para la mayoría de las etapas del procedimiento a la vez que reduce significativamente la radiación secundaria.

- Utilizar fluoroscopia pulsada a la frecuencia más baja posible como default: por ejemplo 7 o 15 cps en lugar de 25 o 30.

- Reemplazar la adquisición con cine digital por fluoroscopia pulsada a baja frecuencia puede reducir la dosis entre cuatro a 10 veces.

- Utilizar la menor magnificación posible y colimar sistemáticamente para reducir el campo a lo estrictamente necesario. Asegurar la mínima distancia entre el detector y el paciente y la mayor distancia entre el tubo de rayos $\mathrm{X}$ y la mesa.

- Evitar las proyecciones más extremas (como OAI caudal). Tratar de evitar la tendencia a realizar todo el procedimiento en solo dos proyecciones ortogonales y rotar las mismas siempre que sea posible para reducir la dosis en el sitio de ingreso del haz de rayos (air kerma) y los efectos sobre la piel.
Estas medidas de técnica radiológica requieren un compromiso activo por parte del médico y técnico durante el procedimiento para ser efectivas y adaptarse a cada etapa. Como ejemplo, es posible que al momento de atravesar la oclusión con la guía en una coronaria derecha en proyección $\mathrm{OAD}$, sea necesario un campo más grande, con alta frecuencia en la fluoroscopia pulsada (25 o 30 pps) para evitar la distorsión por el movimiento. Pasada esta etapa crítica se puede volver rápidamente a los criterios más restrictivos.

\section{Monitoreo y reporte de dosis}

- El límite de radiación tolerable para un procedimiento debe ser expresado en dosis de radiación en lugar de tiempo de fluoroscopia, ya que esta última no incluye la radiación durante la adquisición con cine. Por esto es necesario monitorear en forma permanente la dosis en el sitio de entrada (air kerma): 8 a 10 Gy es el límite de radiación tolerado y a partir de 5 Gy debe informarse al paciente los riesgos/beneficios de continuar ${ }^{13,86}$.

- Dado que con frecuencia son necesarios más de un procedimiento, ya sea por tratamiento de otros vasos o segundo intento en la misma OTC, es importante reportar en el informe o historia clínica tanto la dosis total de radiación (producto dosis/área en grays $/ \mathrm{m}$ ) como la dosis en el punto de ingreso (Air Kerma en grays).

\section{Contraste}

La complejidad y duración de los procedimientos de OTC pueden requerir el uso de gran cantidad de material de contraste. El volumen de contraste yodado es, junto al antecedente de diabetes y de insuficiencia renal preexistente, uno de los tres predictores más importantes para el desarrollo de nefropatía inducida por contraste (NPC) ${ }^{93,94}$. Esta a su vez, aunque suele revertir completamente dentro de los tres meses, es un importante predictor de muerte y deterioro renal progresivo ${ }^{95}$.

\section{RECOMENDACIONES GENERALES PARA REDUCIR LA INCIDENCIA DE NPC EN TODOS LOS PACIENTES}

- Identificar a los pacientes en riesgo de desarrollar NPC. La edad avanzada, deterioro de función renal previa y la diabetes conforman las principales variables pronósticas. La utilización de scores de riesgo (como el Mehran score $^{96}$ ) puede ayudar a extremar en estos casos las medidas de prevención.

- Hidratación. Aunque el protocolo ideal no está claramente establecido, $1 \mathrm{ml} / \mathrm{kg} / \mathrm{h}$ de solución fisiológica a $0,9 \%$ por 24 hs., comenzando 12 hs previo al procedimiento podría ser más efectivo que la SF a $0,45 \%$ y la hidratación en bolo durante el procedimiento o la hidratación ora ${ }^{97}$.

- Preestablecer el monto máximo de contraste razonable para cada paciente, en relación a su función re- 
nal. Como regla general: cuatro veces la tasa de filtrado glomerular ${ }^{98}$. Así, para un paciente con función renal normal el volumen máximo de contraste no debería exceder los $400 \mathrm{ml}$.

- Utilizar contraste no iónico de baja osmolaridad y mantener estabilidad hemodinámica para mantener adecuada perfusión renal.

\section{RECOMENDACIONES PARA REDUCIR LA UTILIZACIÓN DE CONTRASTE EN EL CONTEXTO DE OTC}

- Utilizar catéteres guía y catéteres para inyección contralateral del diámetro menor posible.

- Utilizar inyecciones a través del microcatéter retrógrado para la evaluación de colaterales, en lugar de inyección contralateral.

- Utilizar una guía 0,014 en el lecho distal en posición retrógrada como referencia para reducir controles con material de contraste.

- Utilizar IVUS para guiar el procedimiento una vez atravesada la lesión y predilatado y para redireccionar la guía hacia la luz verdadera en disecciones anterógradas o CART y reverse CART.

- Preferir acceso retrógrado (que requiere menor cantidad de contraste) en caso de pacientes en riesgo de NPC.

\section{PREVENCIÓN Y MANEJO DE COMPLICACIONES EN ANGIOPLASTIA A OCLUSIONES TOTALES CRÓNICAS}

La angioplastia en oclusiones totales crónicas (OTC) constituye uno de los escenarios más desafiantes de la cardiología intervencionista. Esto trae aparejado una tasa de complicaciones mayor respecto a las angioplastias de rutina. Sin embargo, con el advenimiento de materiales dedicados y una adecuada curva de aprendizaje tanto en la prevención como en el tratamiento de las complicaciones esta ha ido disminuyendo, llegando en la actualidad a ser las complicaciones mayores cercanas al $0,5 \% 99$.

Las complicaciones agudas relacionadas a angioplastias en OTC pueden dividirse en cardíacas y extracardíacas. Las extracardíacas son la nefropatía por contraste y la injuria por radiación, las que fueron tratadas en otra sección. Abordaremos aquí las complicaciones cardíacas agudas que son la perforación o ruptura coronaria, las complicaciones isquémicas por injuria de los vasos coronarios, la disección aórtica y el atrapamiento de materiales.

\section{Perforación coronaria}

Constituye una de las complicaciones más graves ya que en muchos casos, de no ser tratada rápida y adecuadamente, conduce al taponamiento cardíaco. Otra consecuencia de la perforación coronaria es el hematoma miocárdico, especialmente en las perforaciones de ramos septales. La incidencia va del 3 al 12\% aproximadamente, depen- diendo de la experiencia de los centros, por lo que es mandatorio su prevención y contar con el entrenamiento y materiales adecuados para su tratamiento. ${ }^{1}$

Las perforaciones coronarias fueron clasificadas por el Dr. S. Ellis en grado I: orificio extraluminal sin tinción miocárdica ni extravasación o disección evidente (hematoma adventicial), grado II: orificio con tinción miocárdica o pericárdica sin extravasación y grado III: orificio con extravasación (la extravasación a una cavidad cardíaca constituye una variante más benigna). Las perforaciones grado I y II suelen asociarse a una evolución favorable (si bien el hematoma septal puede causar estenosis subaórtica), mientras que la tipo III requiere de mayor atención y es a la que nos referiremos en lo sucesivo ${ }^{100}$.

Las perforaciones ocasionadas por las cuerdas guías suelen ser pequeñas y frecuentemente autolimitadas, sin embargo, el riesgo de complicaciones aumenta conforme sucedan eventos que incrementen el tamaño del mismo. Esos sucesos que pueden incrementar el orificio ocasionado por la cuerda guía son el avance de un microcatéter, un balón, un stent, o bien también puede ser consecuencia de la inyección anterógrada de contraste debido a la expansión hidráulica. Otro mecanismo de perforación o ruptura coronaria está mediado por la dilatación de la arteria con el balón, ya que frecuentemente estos vasos presentan importante calcificación y remodelado negativo.

Los pacientes con cirugía cardíaca previa constituyen un grupo con menor incidencia de taponamiento debido a las adherencias del pericardio.

La mejor estrategia para evitar las perforaciones y sus consecuencias es la planificación adecuada del caso, reconociendo estructuras anatómicas predisponentes (circulación en puente, ramos laterales y tortuosidad) y evitando el uso de drogas que puedan agravar la situación (bivalirudina, inhibidores de la GP IIbIIIa). La presencia de una oclusión proximal (ostial) o el uso de catéteres de mayor tamaño pueden generar el "damping" de presiones, de modo tal que la inyección rápida de contraste puede originar por sí mismo la disección coronaria y eventualmente la perforación. Es por esto que se recomienda limitar al máximo las inyecciones anterógradas y de ser necesario realizar inyecciones mínimas con muy poco contraste y de modo lento.

La gran mayoría de las perforaciones coronarias son generadas por la cuerda guía. Dentro del gran espectro de cuerdas utilizadas, representan mayor riesgo de perforación las cuerdas hidrofílicas (por menor sensibilidad manual), las que presentan extremos cónicos adelgazados (tapered) y aquellas de mayor rigidez. Generalmente, estas cuerdas utilizadas para cruzar la entrada a la oclusión (cap) suelen ser modeladas con una mínima curvatura, lo que las hace más agresivas aún. Para minimizar el riesgo de perforación, estas cuerdas no deben ser utilizadas para llegar hasta el sitio de oclusión, sino que se debe llegar con una cuerda blanda atraumática y luego intercambiar mediante el uso de microcatéter por la guía de cruce más agresiva. Una vez en la oclusión el avance debe ser lento y con- 
trolado, visualizando su progresión con proyecciones ortogonales y mediante la visualización con inyecciones retrógradas (contralateral). Inmediatamente que estas cuerdas hayan logrado su propósito deben ser intercambiadas mediante el uso de microcatéteres a cuerdas menos agresivas. En este sentido, es importante remarcar que las cuerdas más agresivas pueden ser sólo necesarias para cruzar la entrada (cap proximal) y no toda la extensión de la oclusión, la cual en su segmento medio suele presentar menos resistencia pudiendo ser atravesadas con cuerdas más seguras. Ante la certeza de estar avanzando en el espacio subintimal y decidir continuar de este modo (técnica de disección y reentrada), el uso de cuerdas blandas hidrofílicas avanzadas de modo prolapsado (knukled wire) constituye la alternativa más segura para evitar perforaciones.

Como fuera mencionado previamente, la perforación coronaria por la cuerda guía suele ser una situación fácilmente controlable, pero debe tenerse especial atención al avance del microcatéter ya que de encontrarse la cuerda guía coronaria en una perforación, el avance del microcatéter tornará la situación en una más riesgosa. Por lo tanto, debe prestarse tanta atención al momento del avance de microcatéteres como al avance mismo de la cuerda guía.

En los casos de avance retrógrado, existe la posibilidad de perforación de los vasos que otorgan circulación colateral (vasos "donantes"). Como fuera mencionado, esta reviste menos gravedad en ramos intramiocárdicos (ramos septales) respecto a los vasos epicárdicos, por lo que es aconsejable el avance por esta última vía luego de una adecuada curva de aprendizaje. En algunos casos, la ruptura coronaria no la ocasiona la cuerda guía sino el microcatéter, principalmente en casos de circulación colateral con extrema tortuosidad. Habitualmente, la perforación de estos ramos suele ser evidente una vez retirado el microcatéter al finalizar el procedimiento, por lo que es recomendable al retirar el microcatéter dejar la cuerda guía retrógrada con su porción blanda a nivel de la circulación colateral y realizar una angiografía de control previo al retiro de la cuerda, manteniendo así la posibilidad de algún tratamiento frente a la eventualidad de una ruptura coronaria.

El tratamiento de la ruptura coronaria varía fundamentalmente en relación al sitio en el que ocurra, ya sea en el vaso tratado en sitios cercanos a la oclusión, en el vaso tratado o sus ramos laterales a nivel distal o terminal o en la circulación colateral en casos de acceso retrógrado.

a. Ruptura del vaso tratado en sitios cercanos a la oclusión. Para el tratamiento de este tipo de rupturas la primera maniobra que debe realizarse siempre es la insuflación de un balón oclusivo a nivel proximal del sitio de ruptura o en el mismo sitio de ruptura con la intención de parar el sangrado. Este balón debe dejarse insuflado en forma prolongada y reiteradamente de persistir la fuga de contraste. Esta maniobra suele ser efectiva y suficiente en la mayoría de los casos, caso contrario, debe realizarse el implante de un stent cubierto (stent graft) en el sitio de la perforación ${ }^{101}$. En casos de rupturas con gran sangrado se sugiere im- plantar el stent cubierto con técnica de doble catéter (técnica de ping-pong) la que consiste en avanzar el stent cubierto por un segundo catéter guía mientras se mantiene insuflado el balón que frena el sangrado, desinflando este sólo para permitir el avance de la segunda cuerda y luego del stent cubierto. Los catéteres guía serán introducidos en la coronaria o retirados hacia la aorta en forma alternada según necesidad. [102]

b. Ruptura del vaso tratado o sus ramos laterales a nivel distal. Esta ruptura es ocasionada por una cuerda guía agresiva (hidrofílica o con alto índice de penetrancia). Esta perforación puede prevenirse mediante la inyección dual para evitar el avance distal en ramos secundarios sumado al inmediato intercambio de la cuerda guía por una más atraumática luego del cruce de la oclusión. Para su diagnóstico es importante abarcar en el control angiográfico el segmento terminal del vaso tratado y sus ramos, prestando especial atención a las imágenes ya que puede pasar desapercibido. Nuevamente aquí la maniobra inicial debe ser el insuflado de un balón de modo que este sea oclusivo en forma prolongada y reiteradamente de persistir la fuga de contraste. Por lo general esta maniobra logra hacer hemostasis, de lo contrario debe avanzarse un microcatéter bien distal cercano al sitio de la perforación y luego de retirada la cuerda guía se realiza la oclusión del vaso mediante el implante de coils o por medio de la embolización de grasa subcutánea ${ }^{103,104}$ En estos casos no es mandatorio revertir la anticoagulación, ya que de poder controlarse, es factible continuar con la angioplastia en caso que no haya sido finalizada al momento del diagnóstico de la perforación.

c. Ruptura de vasos donantes (circulación colateral). Este escenario ocurre en el marco de angioplastia por acceso retrógrado. Como ya fuera mencionado, la perforación de ramos intramiocárdicos (ramos septales) se asocian a mejor evolución, si bien se debe estar atento a que grandes hematomas septales pueden ocasionar la presencia de gradiente subvalvular, trastornos de conducción eléctrica o bien evolucionar a la ruptura cardíaca $(\mathrm{CIV})^{105,106}$. Contrario a esto, la perforación de vasos donantes epicárdicos pueden derivar en derrame pericárdico y taponamiento cardíaco. Su tratamiento es más complejo y requiere de experiencia. La primera estrategia descripta es la de avanzar el microcatéter proximal al sitio de la perforación y realizar aspiración a través del mismo. Esto genera el colapso del vaso colateral y en muchos casos se logra controlar el sangrado. Si esto no fuera suficiente se deberá proceder a la exclusión del vaso, la cual será diferente en la medida que la oclusión crónica haya sido resuelta o no. Si la oclusión no ha sido resuelta, la liberación de coil desde el vaso donante será suficiente, por el contrario, si la oclusión crónica 
ha sido resuelta exitosamente se deberá liberar coil desde el vaso donante y el vaso receptor para evitar el flujo sanguíneo en ambos sentidos. Si estas medidas de hemostasia no pudieran ser realizadas o no fueran suficientes se deberá considerar la cirugía cardíaca de urgencia.

En vista de lo antedicho resulta evidente que los centros que realicen oclusiones totales crónicas deberán tener rápidamente disponible en la sala de hemodinamia un kit de pericardiocentésis, stent cubiertos (stent graft) de diversos tamaños, coils, así como contar con la posibilidad de realizar ecocardiografía de urgencia en la sala de hemodinamia.

\section{Complicaciones isquémicas}

Las complicaciones isquémicas pueden ocurrir por injuria del vaso tratado, del vaso donante o de la circulación colateral, estas últimas en el caso de acceso retrógrado.

a. Injuria del vaso tratado. Es importante considerar que si bien el vaso a tratar se encuentra previamente ocluido, es posible que proximal a la oclusión la arteria de origen a ramos secundarios o bien sea fuente de ramos colaterales, los cuales podrían verse comprometidos frente al daño del vaso. La causa más común de injuria del vaso tratado es la disección coronaria, la cual puede suceder por el manipuleo de las cuerdas guía coronaria, la inyección agresiva de contraste anterógrado, la utilización de catéter guía en forma agresiva o el daño por el uso de extensores de catéter guía (técnica de mother and child $)^{107}$. Considerando estas potenciales causas de disección del vaso es lógico comprender las estrategias para su prevención, entre las que se destacan el uso cauteloso de catéter guía, evitando el damping de presiones y controlando el volumen y intensidad de las inyecciones de contraste. Por otro lado, siempre deben utilizarse cuerdas guías blandas y sistema de microcatéter para alcanzar el sitio de la oclusión, evitando el paso de materiales más agresivos por los segmentos proximales del vaso. Los extensores de catéter guía no deben avanzarse en forma agresiva, de ser necesaria la dilatación con balón esta deberá realizarse, progresando luego el extensor sobre el soporte del balón. Las disecciones coronarias ocasionadas por el manipuleo del catéter guía o el daño hidráulico de la inyección agresiva pueden comprometer el segmento aorto-coronario y evolucionar hacia la disección de la aorta la cual generalmente compromete en el inicio el seno coronario y luego progresar hacia la aorta ascendente. Una mención especial merece la técnica de disección y reentrada, ya que en este caso la disección se genera como parte de la técnica, siendo por lo tanto muy importante limitar la disección únicamente al segmento de la oclusión, evitando así comprometer innecesariamente ramos funcionantes. El tratamiento de la in- juria del vaso tratado requiere por lo general del implante de stent, siendo necesario en el caso de disección aortocoronaria el implante cubriendo completamente el ostium coronario.

b. Injuria del vaso donante. Esta complicación puede suceder más frecuentemente en la técnica de revascularización por vía retrógrada. El daño del vaso donante suele ser de extrema gravedad y potencialmente mortal ya que por lo general este vaso irriga un gran territorio miocárdico (el propio y el del vaso ocluido por colaterales). Las principales causas de injuria del vaso donante son las disecciones mediadas por el manipuleo del catéter, por el avance de materiales hacia el vaso receptor o por trombosis. Para prevenir la trombosis se sugiere un monitoreo frecuente del estado de coagulación (ACT), ya que por lo general estas prácticas son prolongadas y requieren de administración adicional de Heparina durante el procedimiento. La disección requerirá de la urgente reparación mediante el implante de stent, lo que a veces puede ser complejo por la presencia de materiales (cuerdas y catéteres) transcurriendo hacia el vaso receptor.

c. Injuria de la circulación colateral. La oclusión de ramos colaterales pueden darse secundariamente al pasaje de la cuerda guía o el microcatéter durante procedimientos de revascularización por vía retrograda. La oclusión de estos vasos también puede ser consecuencia del tratamiento con coils debido a la presencia de una perforación ${ }^{108}$. La circulación colateral epicárdica suele ser mas tortuosa y es más factible de ser dañada, por lo que a modo de prevención de esta complicación se recomienda el uso de cuerdas guías con puntas extremadamente atraumáticas, evitar forzar el avance de dispositivos y dejar el acceso por colaterales epicárdicas como última opción ${ }^{85}$.

\section{Atrapamiento de materiales}

Dada las características de la anatomía coronaria en las OTC existe un riesgo aumentado de atrapamiento de los materiales en las arterias coronarias, aunque podría decirse que la probabilidad de esta complicación es relativamente baja, siendo aproximadamente del 0,3\% ${ }^{109}$.

a. Atrapamiento de la cuerda guía coronaria: la cuerda guía puede quedar atrapada al atravesar placas con alto contenido cálcico, como consecuencia del pasaje en sitios de extrema tortuosidad o por el "anudamiento" producto de una excesiva rotación. Para su extracción la maniobra recomendada es el avance de un microcatéter hasta el sitio del atrapamiento y luego intentar ingresar la cuerda dentro del microcatéter para su extracción. La maniobra debe ser lenta y cuidadosa para evitar la fractura de la cuerda o el avance agresivo del catéter guía hacia la arteria coronaria.

b. Atrapamiento de microcatéteres o balones: El atrapamiento del microcatéter o de un balón dentro del vaso coronario requiere de maniobras controladas 
para evitar la ruptura o perforación del vaso. Para ello, es recomendable cortar el conector proximal y avanzar cuidadosamente un catéter (o un extensor de catéter guía) con un lazo en el interior abrazando el cuerpo (shaft) del balón o microcatéter. Una vez avanzado lo más distalmente posible traccionar del lazo en forma controlada el material atrapado intentando capturarlo dentro del catéter o extensor de catéter para su posterior retiro. De ser posible se recomienda mantener una cuerda en paralelo para el tratamiento de una eventual ruptura del vaso.

\section{MATERIALES NECESARIOS PARA RESOLUCIÓN DE COMPLICACIONES EN EL TRATAMIENTO DE OTC}

\author{
1. Disponibilidad de ecocardiografía en la Sala de \\ Hemodinamia.
}

2. Set de pericardocentesis

3. Variedad de cuerdas guía coronaria y microcatéteres.

4. Stent cubiertos (stent graft) con variedad de medidas.

5. Coils.

6. Lazo coronario.

\section{REFERENCIAS}

1. Kahn JK. Angiographic suitability for catheter revascularization of total coronary occlusions in patients from a community hospital setting. Am Heart $J$ 1993; 126(3): 561-4.

2. Olivari Z, Rubartelli P, Piscione F, et al. Immediate results and one-year clinical outcome after percutaneous coronary interventions in chronic total occlusions: data from a multicenter, prospective, observational study (TOAST-GISE). Journal of the American College of Cardiology. J Am Coll Cardiol 2003; 41(10): 1672-8.

3. Claessen BEPM, van der SchaafRJ, Verouden NJ, et al. Evaluation of the Effect of a Concurrent Chronic Total Occlusion on Long-Term Mortality and Left Ventricular Function in Patients After Primary Percutaneous Coronary Intervention. J Am Coll Cardiol Intv 2009; 2(11): 1128-34.

4. FeferP, Knudtson ML, Cheema AN, et al. Currentperspectives on coronary chronic total occlusions: the Canadian Multicenter Chronic Total Occlusions Registry. J Am Coll Cardiol. 2012:59:991-7.

5. King SB, Aversano T, Ballard WL, et al. ACCF/AHA/SCAl 2007 update of the clinical competence statement on cardiac interventional procedures a report of the American College of Cardiology Foundation/American Heart Association/ American College of Physicians Task Force on Clinical Competence and Training. J Am Coll Cardiol 2007; 50: 82-108

6. Di Mario C, Werner GS, Sianos G, et al. European perspective in the Recanalisation of Chronic Total Occlusions (CTO): consensus document from the EuroCTOClub. Eurolntervention. 2007;3:30-43.

7. Thompson CA, Jayne JE, Robb JF, et al. Retrograde Techniques and the Impact of Operator Volume on Percutaneous Intervention for Coronary Chronic Total Occlusions: An Early U.S. Experience. J Am Coll Cardiol Intv 2009; 2(9): 834-42.

8. Habara M, Tsuchikane E, Muramatsu T, et al. Comparison of percutaneous coronary intervention for chronic total occlusion outcome according to operator experience from the Japaneseretrograde summitregistry. Catheter Cardiovasc Interv. 2016 May;87(6):1027-35.

9. MaielloL, Colombo A, Gianrossi R, etal. Coronaryangioplasty of chronicocclusions: Factors predictive of procedural success. Am Heart J 1992; 124(3): 581-4.

10. Giokoglu K, Preusler W, Störger H, et al. The recanalization of chronic coronary artery occlusions: what factors influence success?. Dtsch Med Wochenschr 1994; 119(51-52): 1766-70.

11. Surmely J-F, Tsuchikane E, Katoh O, et al. New concept for CTO recanalization using controlled antegrade and retrograde subintimal tracking: the CART technique. J Invasive Cardiol 2006; 18(7): 334-8.

12. Stecker MS, Balter S, Towbin RB, et al. Guidelines for Patient Radiation Dose Management. J Vasc Interv Radiol 2009; 20(7): S263-73.

13. Brilakis ES, Grantham JA, Rinfret S, et al. A percutaneous treatment algorithm for crossing coronary chronic total occlusions. JACC Cardiovasc Interv. 2012; 5:367-79.

14. Karmpaliotis D, Lembo N, Kalynych A, et al. Development of a high-volume, multiple-operator program for percutaneous chronic total coronary occlusion revascularization:procedural, clinical, and cost-utilization outcomes. Catheter Cardiovasc Interv. 2013;82:1-8.

15. RinfertS. Percutaneuos Intervention for Coronary Chronic TotalOcclusions. The hybrid approach. Editorial Sringer, 2016.

16. Sathananthan J, Dzavik V. Coronary intervention for chronic total occlusion, current indications and future directions. Coronary Artery Disease 2017,28:426-436.

17. Azzlini L, Jolicoeur EM, Pighi M et al. Epidemiology, management strategies, and outcomes of patients with chronic total coronary occlusion. Am J cardiol 2016,118;1128-1135.

18. Yamamoto E, Natsuaki M, Morimoto T, et al. Long Term outcomes after Percutaneous Coronary intervention for Chronic Total Occlusion (from the CREDO Kyoto registry). Am J Cardiol 2013,112:767-74.

19. Bardaji A, Rodriguez-Lopez J, Torres-Sanchez M. Chronic Total Occlusions: To treat or not to treat. World J Cardiol 2014;6(7):621-629.

20. Brilakis ES, Abdullah SM, Banerjee S. Who should undergo chronic total occlusion percutaneous coronaryintervention? Theexploration continues. JAm Coll Cardiol 2016:68:1633-1636.

21. Werner GS, Surber R, Ferrari M, et al. The functional reserve of collaterals supplying long-term chronic total coronary occlusions in patients without prior myocardial infarction. Eur Heart J 2006,27:2406-2412.

22. Safley DM, Koshy S, Grantham JA, et al. Changes in myocardial ischemic burdenfollowing percutaneous, coronary intervention of chronictotal occlusions. Catheter Cardiovasc Interv 2011;78:337-343.

23. Galassi AR, Werner GS, Tomasello SD et al. Prognostic value of exercise myocardial scintigraphy in patients with coronary chronic total occlusions. J Interv Cardiol 2010;23:139-148.

24. JoyalD, Afilalo J, Rinfret S. Effectiveness of racanalization of chronic total occlusions: asystematic review and meta-analysis. Am Heart J 2010;160(1):179-87.

25. Grantham JA, Jones PG, Cannon L, et al. Quantifying the early health status benefits of succesfull chronic total occlusions recanalization: results from the FlowCardia's Approach to Chronic Total Occlusions Recanalization (FACTOR) Trial. Circ Cardiovasc Qual octcomes 2010:3:284-90.

26. Sapontis J, Salisbury AC, Yeh RW, et al. Early Procedural and Health Status Outcomes After Chronic Total Occlusion Angioplasty: A report From the OPENCTORegistry (Outcomes, Patient Health Status, and Efficiency in Chronic Total Occlusion Hybrid Procedures). J Am Coll Cardiol Intv 2017;10(15):1523-1534.

27. Hoebers LP, Claessen BE, Elias J, et al. Meta-analysis on the impact of percutaneous coronary interventions of chronic total occlusions on left ventricular function and clinical outcomes. Int J Cardiol 2015;187;90-96.

28. Henriques JPS, Hoebers LP, Ramunddal T, et al. Percutaneous Intervention for Concurrent Chronic Total Occlusions in Patients With STEMI. The EXPLORE Trial. J Am Coll Cardiol 2016;68:1622-1632.

29. Choi IJ, Koh YS, Lim S, et al. Impact of Percutaneous Coronary Intervention for Chronic Total Occlusion in non-infarct-related arteries in Patients with Acute Myocardial Infarction (from COREA-AMI Registry). Am J Cardiol 2016;117(17):1039-1046

30. GerogeS, Cockburn J, Clayton TC, et al. Long-Term follow up of elective chronic total occlusions angioplasty: Analysis from the UK Central Cardiac AuditDatabase. J Am Coll Cardiol 2014;64:235-243.

31. Motoma S. et al. Int J Cardiol 2017

32. Patel MR, Dehmer GJ, Hirshfeld JW, et al. Appropiate use criteria for coronary revascularization. J Am Coll Cariol 2012;59:857-81.

33. Windecher S, KolhP, Alonso F, et al. Guidelines on myocardial revascularization. Eur Heart J 2014;35(37):2541-619.

34. Rangan BV, Kostia A, Christopoulos G, et al. The Hybrid Approach to Intervention of Chronic Total Occlusions. Curr Cardiol Rev 2014;11(4):299-304.

35. Singh $M$, Bell MR, Berger PB, et al. Utility of bilateral coronary injections during complex coronary angioplasty. J Invasive Cardiol 1999;11:70-74. 
36. Morino Y, Abe M, Morimoto T, et al. Predicting successful guidewire crossing through chronic total occlusion of native coronary lesions within 30 minutes: the J-CTO (Multicenter CTORegistry in Japan) score as a difficulty grading and time assessment tool. JACC Cardiovasc Interv 2011;4(2):213-221.

37. Maeremans J, Spratt JC, Knaapen P, et al. Towards a Contemporary, Comphensive Scoring System for Determing Technical Outcomes of Hybrid Percutaneous Chronic Total Occlusion Treatment: The RECHARGE Score. Catheter Cardiovasc Interv 2017.

38. Katsuragawa M, Fujiwara H, MiyamaneM, et al. Histologic Studies in Percutaneous Transluminal Coronary Angioplasty for Chronic Total Occlusion: Comparison of Tapered and Abrupt Types of Occlusion and Short and Long Occluded Segments. J Am Coll Cardiol 1993;21:604-611.

39. Srivatsa SS, Edwards WD, Boos CM, et al. Histologic Correlates of Angiographic Chronic Total Coronary Artery Occlusions. Influence of Occlusion Duration on Neovascular Channel Patterns and Intimal Plaque Composition. J Am Coll Cardiol 1997;29:955-963.

40. Irving J. CTO pathology, how does this affect management. Curr Cardiol Rev. 2014;10(2):99-107.

41. Spratt JC, Wilson WM. The treatment of chronic total occlusions: advances in procedural techniques - antegrade. Curr Cardiol Rev 2014;31.

42. Muhammad Kl, Lombardi WL, Christofferson R, et al. Subintimal guidewire tracking during successful percutaneous therapy for chronic coronary total occlusions: insights from an intravascular ultrasound analysis. Catheter Cardiovasc Interv 2012;79(1):43-48

43. Kotsia A, Christopoulos G, Brilakis ES. Use of the retrograde approach for preserving the distal bifurcation after antegrade crossing of a right coronary artery chronic total occlusion. J Invasive Cardiol 2014; 26: E48-9.

44. PittB. Interarterial coronary anastomoses. Occurrence in normal hearts and in certain pathologic conditions. Circulation 1959;20:816-822.

45. BacheRJ,SchwartzJS:Myocardial bloodflow during exercise after gradual coronary occlusion in the dog. Am J Physiol 1983, 245:H131-H138.

46. Rentrop KP, Cohen M, BlankeH, Phillips RA:Changes in collateral channel filling immediately after controlled coronary artery occlusion by an angioplasty baIloon in human subjects. J Am Coll Cardiol 1985, 5:587-592.

47. Werner GS, Ferrari M, HeinkeS, et al. Angiographic assessment of collateral connections in comparison with invasively determined collateral function in chronic coronary occlusions. Circulation. 2003;107:1972-7.

48. Meier P, Hemingway $H$, Lansky $A$, et al: The impact of the coronary collateral circulation on mortality: a meta-analysis. Eur Heart J 33(5):614-621, 2012.

49. McEntegart MB, Badar AA, Ahmad FA, et al. The collateral circulation of coronary chronic total occlusions. Eurointervention 2016;11:e1596-e1603.

50. Sianos G, Barlis P, Di Mario C et al. EuroCTO Club. European experience with the retrograde approach for the recanalization of coronary artery chronic total occlusions. A report on behalf of the EuroCTO Club. Eurolntervention 2008;4(1):84-92.

51. Rathore S, Katoh O, Matsuo H et al. Retrograde percutaneous recanalization of chronic total occlusion of the coronary arteries: procedural outcomes and predictors of success in contemporary practice. Circ. Cardiovasc. Interv 2009;2(2):124-132.

52. Mogabgab O, Patel VG, Michael TT, et al. Long-term outcomes with use of the crossboss and stingray coronary cto crossing and reentry devices. J Invasive Cardiol 2013; 25: 579-85.

53. Godino C, Latib A, Economou Fl, et al. Coronary chronic total occlusions: midterm comparison of clinical outcome following the use of the guided-STAR technique and conventional anterograde approaches. Catheter Cardiovasc Interv 2012; 79: 20-7.

54. Christopoulos G, Menon RV, Karmapallotis D, et al. The efficacy and safety of the "Hybrid" Approach to coronary chronic total occlusions: Insights from a Contemporary Multicenter US Registry and Comparison with Prior studies. J Invasive Cardiol 2014;26(9):427432.

55. Maeremans J, Dens J, Spratt JC, et al. Antegrade Dissection and Reentry as Part of the Hybrid Chronic Total Occlusion Revascularization Strategy: A Subanalysis of the RECHARGERegistry (Registry of CrossBoss and Hybrid Procedures in France, the Netherlands, Belgium and United Kingdom). Circ Cardiovasc Interv 2017;10(6).

56. Harding SA, WU EB, LO S, et al. A new algorithm for crossing chronic total occlusions from the Asia Pacific chronic total occlusion club. J Am Coll Cardiol Intv 2017;10:2135-2143.

57. GalassiAR, TomaselloSD, ReifartN, etal.In-hospital outcomes of percutaneous coronaryintervention in patients with chronic totalocclusion: insights from the ERCTO registry. Eurointervention.2011;7:472-9
58. Furuichi S, Airoldi F, Colombo A. Intravascular ultrasound-guided wiring for chronic total occlusion. Catheter Cardiovasc Interv 2007;70:856-9.

59. Ito S, Suzuki T, Ito T, et al. Novel technique using intravascular ultrasound-guided guidewire cross in coronary intervention for uncrossable chronic total occlusions. Circ J 2004;68:1088-92.

60. Park Y, Park HS, Jang GL, et al. Intravascular ultrasound guided recanalization of stumpless chronic total occlusion. Int J Cardiol 2011;148:174-8.

61. Rathore S, Katoh O, Tuschikane E, et al. A novel modification of the retrograde approach for the recanalization of chronic total occlusion of the coronary arteries intravascularultrasound-guided reverse controlled antegrade and retrograde tracking. JACC Cardiovasc Interv 2010;3:155-64.

62. Chieffo A, Latib A, Caussin C, et al. A prospective, randomized trial of intravascular-ultrasound guided compared to angiography guided stent implantation in complex coronary lesions: the AVIO trial. Am Heart J 2013;165:65-72.

63. HongSJ, Kim BK, Shin DH, et al. Usefulness of intravascular ultrasound guidance in percutaneous coronary intervention with second-generation drug-eluting stents for chronic total occlusions (from the Multicenter Korean-Chronic Total Occlusion Registry). Am J Cardiol 2014;114:534-40.

64. Tian NL, Gami SK, Ye F, et al. Angiographic and clinical comparisons of intravascular ultrasound-versus angiography-guided drug-eluting stent implantation for patients with chronic total occlusion lesions: two-year results from a randomised AIR-CTO study. Eurolntervention 2015;10:1409-17.

65. Kim BK, Shin DH, Hong MK, et al. Clinical impact of intravascular ultrasound-guided chronic total occlusion intervention with zotarolimus-eluting versus biolimus-eluting stent implantation: randomized study. Circ Cardiovasc Interv 2015;8:e002592.

66. Song L, Maehara A, Finn MT, et al. Intravascular Ultrasound Analysis of Intraplaque versus Subintimal Tracking in Percutaneous Intervention for Coronary Chronic TotalOcclusions and Association with ProceduralOutcomes. JAm Coll Cardiol 2017;10:1011-1021.

67. Wilson WM, Hanratty C, Walsh S, EgredM, McEntagertM, OldroydK. Strange J, SprattJC. Outcomes from the UKCTOHybrid database.Presented@ TCT.2014.

68. Pershad A, Eddin M, Girotra S, Cotugno R, Daniels D, Lombardi W. Validation and incremental value of the hybrid algorithm for CTO PCI. Catheter Cardiovasc Interv. 2014;84(4):654-9.

69. Brilakis ES, Karmpaliotis D, Werner GS, et al. Developments in coronary chronic total occlusion percutaneous coronary interventions: 2013 state-of- theart update. J Invasive Cardiol. 2014;26(6):261-6.

70. Reifart N, Enayat D, Giokoglu K. A novel penetration catheter (Tornus) as bailout device after balloon failure to recanalise long, old calcifi ed chronic occlusions. Eurolntervention. 2008;3(5):617-21.

71. Whitlow PL, Burke N, Lombardi WL, et al. Use of a novel crossing and re-entry system in coronary chronic total occlusions that have failed standard crossing techniques. JACC Cardiovasc Interv 2012;5:393-401.

72. Soon KH, Cox N, Wong A, Chaitowitz I, Macgregor L, Santos PT, et al. CT coronary angiography predicts the outcome of percutaneous coronary intervention of chronic total occlusion. J Interv Cardiol. 2007; 20(5):359-66

73. Galassi AR, Tomasello SD, Costanzo L, Campisano MB, Barrano G, Ueno M, et al. Mini-STAR as bail-out strategy for percutaneous coronary intervention of chronic total occlusion. Catheter Cardiovasc Interv. 2012;79:30-40.

74. Colombo A, Mikhail GW, Michev l, et al. Treating chronic total occlusions using subintimal tracking and reentry: the STAR technique. Catheter Cardiovasc Interv. 2005;64:407-11.

75. Carlino M, Godino C, Latib A, Moses JW, Colombo A. Sub-intimal tracking and re-entry technique with contrast guidance: a safer approach. Catheter Cardiovasc Interv. 2008;72:790-6.

76. Michael TT, Papayannis AC, Banerjee S, Brilakis ES. Subintimal dissection/reentry strategies in coronary chronic total occlusion interventions. Circ Cardiovasc Interv. 2012;5:729-38.

77. Valenti R, Vegara R, Migliorini A, et al. Predictors of reocclusion after successful drug-eluting stent-supported percutaneous coronary intervention of chronic total occlusion. J Am Coll Cardiol. 2013;61:545-50

78. Rinfret $S$, Ribeiro HB, Nguyen CM, et al. Dissection and re-entry techniques and longer-term outcomes following successful percutaneous coronary intervention of chronic total occlusion. Am J Cardiol. 2014;114:1354-60.

79. Wilson WM, Walsh SJ, Bagnall A, et al. One-Year Outcomes After Succesful Chronic Total Occlusion Percutaneous Coronary Intervention: The impact of Dissection Re-Entry Techniques. Catheter Cardiovasc Interv 2017.

80. Kahn JK, Hartzler GO. Retrograde coronary angioplasty of isolated arterial segments through saphenous vein bypass grafts. Catheterization and cardiovascular diagnosis. Jun 1990;20(2):88-93. 
81. Christopoulos G, Karmpaliotis D, Alaswad K, et al. Application and outcomes of a hybrid approach to chronic total occlusion percutaneous coronary intervention in a contemporary multicenter US registry. Internationaljournal of cardiology. Nov 01 2015;198:222-228.

82. Tsuchikane E, YamaneM, MutohM, et al. Japanesemulticenterregistryevaluating the retrograde approach for chroniccoronary totalocclusion. Catheterization and cardiovascular interventions: official journal of the Society for Cardiac Angiography \& Interventions. Nov 01 2013;82(5):E654-661.

83. Galassi AR, Sianos G, Werner GS, et al. Retrograde Recanalization of Chronic Total Occlusions in Europe: Procedural, In-Hospital, and Long-Term Outcomes From the Multicenter ERCTO Registry. Journal of the American College of Cardiology. Jun 09 2015;65(22):2388-2400.

84. Karmpaliotis D, Michael TT, Brilakis ES, et al. Retrograde coronary chronic total occlusion revascularization: procedural and in-hospital outcomes from a multicenter registry in the United States. JACC. Cardiovascular interventions. Dec 2012;5(12):1273-1279.

85. JoyalD, Thompson CA, Grantham JA, Buller CE, Rinfret S. The retrogradetechnique for recanalization of chronic total occlusions: a step-by-step approach. JACC. Cardiovascular interventions. Jan 2012;5(1):1-11.

86. Sianos G, Werner GS, Galassi AR. Recanalization of Chronic Total coronary Occlusions: 2012 consensus document from the EuroCTO club. Eurointervention 2012:8:139-145.

87. AlaswadK, MenonRV, Christopoulos G, etal. Transradial approach forcoronary chronic total occlusion interventions: insights from a contemporary multicenter registry. Catheter Cardiovasc Interv 2015;85(7):1123-1129

88. Bakker EJ, Maeremans J, Zivelonghi C, et al. Fully transradial versus transfemoral approach for percutaneous intervention of coronary chronic total occlusions applying the hybrid algorithm. Insights from RECHARGERegistry. Circ Cardiovasc Interv 2017.

89. Dautov R, Ribeiro HB, Abdul-Jawad Altisient O, et al. Effectiveness and Safety of the transradial 8FSheathless Approach for Revascularization of Chronic Total Occlusions. Am J Cardiol 2016;118(6):785-789.

90. Suzuki S1, Furui S, Kohtake H, Yokoyama N, Kozuma K, Yamamoto Y, Isshiki T. Radiation exposure to patient's skin during percutaneous coronary intervention forvarious lesions, including chronic total occlusion. Circ J. 2006;70:44-48.

91. ICRP, 2013. Radiological protection in cardiology. ICRP Publication 120. Ann. ICRP 42(1).

92. Suzuki S., Furui S., Isshiki T., et al. Methods to reduce patients' maximum skin dose during percutaneous coronary intervention for chronic total occlusion. Catheter Cardiovasc Interv (2008)71:792-798

93. Rihal CS, Textor SC, Grill DE, Berger PB, Ting HH, Best PJ, Singh M, Bell MR, Barsness GW, Mathew V, Garratt KN, Holmes JrDR. Incidence and prognostic importance of acute renal failure after percutaneous coronary intervention. Circulation. 2002; 105:2259-64.

94. James MT, Ghali WA, Tonelli M, Faris P, Knudtson ML, Pannu N, Klarenbach SW, Manns BJ, Hemmelgarn BR. Acute kidney injury following coronary angiography is associated with a long-term decline in kidney function. Kidney Int. 2010;78:803-19.

95. James MT, Ghali WA, Knudtson ML, Ravani P, Tonelli M, Faris P, Pannu N, Manns BJ, Klarenbach SW, Hemmelgarn BR, Alberta Provincial Project for Outco-
meAssessment in Coronary HeartDisease (APPROACH) Investigators. Associations between acute kidney injury and cardiovascular and renal outcomes after coronary angiography. Circulation. 2011;123:409-16.

96. Mehran R, Aymong ED, Nikolsky E, Lasic Z, lakovou I, Fahy M, Mintz GS, Lansky AJ, Moses JW, Stone GW, Leon MB, Dangas G. A simple risk score for prediction of contrast-induced nephropa-thy after percutaneous coronary intervention: development and ini- tial validation. J Am Coll Cardiol. 2004:44:1393-9.

97. Bader BD, BergerED, Heede MB, Silberbaur I, Duda S, Risler T, Erley CM. What is the best hydration regimen to prevent contrast media-induced nephrotoxicity? Clin Nephrol. 2004;62:1-7.

98. Mager A, Vaknin Assa H, LevEl, Bental T, AssaliA, KornowskiR. The ratio of contrastvolume to glomerularfiltration ratepredicts outcomes afterpercutaneous coronary intervention for ST-segment elevation acute myocardial infarction. Catheter Cardiovasc Interv. 2011 Aug 1;78(2):198-201.

99. Patel VG, Brayton KM, Tamayo A, Mogabgab O, Michael TT, Lo N, et al. Angiographic success and procedural complications in patients undergoing percutaneous coronary chronic total occlusion interventions: a weighted meta-analysis of 18,061 patients from 65 studies. JACC Cardiovasc Interv. 2013;6(2):128-36.

100. Ellis SG, Ajluni S, Arnold AZ, Popma JJ, Bittl JA, Eigler NL, Cowley MJ, Raymond RE, Safian RD, Whitlow PL. Increased coronary perforation in the new device era. Incidence, classification, management, and outcome. Circulation. 1994:90:2725-30

101. Al-Mukhaini M, Panduranga P, Sulaiman K, Riyami AA, Deeb M, Riyami MB. Coronary perforation and covered stents: an update and review. Heart Views. 2011;12(2):63-70

102. Ben-Gal Y, Weisz G, Collins MB, Genereux P, Dangas GD, Teirstein PS, et al. Dual catheter technique for the treatment of severe coronary artery perforations. Catheter Cardiovasc Interv. 2010;75(5):708-12.

103. Oda H, Oda M, Makiyama Y, Kashimura T, Takahashi K, Miida T, et al. Guidewire-induced coronary artery perforation treated with transcatheter delivery of subcutaneous tissue. Catheter Cardiovasc Interv. 2005;66(3):369-74.

104. Ponnuthurai FA, Ormerod OJ, Forfar C. Microcoil embolization of distal coronary artery perforation without reversal of anticoagulation: a simple, effective approach. J Invasive Cardiol. 2007;19(8): E222-5.

105. Lin TH, Wu DK, Su HM, Chu CS, Voon WC, Lai WT, et al. Septum hematoma: a complication of retrograde wiring in chronic total occlusion. Int J Cardiol. 2006:113(2):e64-6.

106. Fairley SL, Donnelly PM, Hanratty CG, WalshSJ. Images in car-diovascularmedicine. Interventricular septal hematoma and ven-tricular septal defect after retrograde intervention for a chronic total occlusion of a left anterior descending coronary artery. Circulation. 2010;122(20):e518-21.

107. Shorrock D, et al. Frequency and outcomes of aortocoronary dis- section during percutaneous coronary intervention of chronic total occlusions: a case series and systematic review of the literature. Catheter Cardiovasc Interv. 2014;84(4):670-5

108. Brilakis ES, et al. The retrograde approach to coronary artery chronic total occlusions: a practical approach. Catheter Cardiovasc Interv. 2012;79(1):3-19.

109. Alomari I, Snider R, Ponce S, Ahmed B. Entrapped devices after pci. CardiovasC Revasc Med. 2014;15:182-5. 